\title{
Consumer Sophistication, Word-of-mouth and "False" Promotions
}

\author{
Yiting Deng Richard Staelin Wei Wang William Boulding*
}

\begin{abstract}
Not all retail price promotions provide consumers with additional monetary value. For instance, a retailer might raise the retail price of an item just before a sale and then "promote" the product by listing the initial price as the sales price. This paper addresses the question "under what conditions would a firm give such a 'false' promotion and with what regularity?" with an analytical model composed of two competing retailers and two segments of consumers. The duopoly firms sell substitutable products in a finite number of periods to consumers. In each period, each firm can choose to offer a real promotion, a false promotion, or no promotion. One segment of consumers is sophisticated and is able to discern false promotions based on the two firms' promotional offerings. The other segment of consumers is naive and trusts each firm's claimed promotional offering. Sophisticated consumers pass information on the existence of false promotions via word-of-mouth (WOM) to a fraction of naive consumers.

We find that the possibility of offering false promotions can improve the payoffs for both firms, especially when the market is more competitive. Moreover, even when consumers can identify that a firm is offering a false promotion and pass this information to others, a firm still finds it optimal to offer such promotions from time to time. In general, the firm is more likely to offer false promotions in an environment with lower competition, fewer sophisticated consumers, and weaker WOM.
\end{abstract}

Keywords: false promotion, word-of-mouth, reference-dependence, game theory

${ }^{*}$ Yiting Deng is an Assistant Professor at the UCL School of Management, University College London (email: yiting.deng@ucl.ac.uk). Richard Staelin is the Gregory Mario and Jeremy Mario Professor of Business Administration at the Fuqua School of Business, Duke University (email: rstaelin@duke.edu). Wei Wang is an Assistant Professor at the University of International Business and Economics (email: weiwang@uibe.edu.cn). William Boulding is the Dean and J.B. Fuqua Professor of Business Administration at The Fuqua School of Business, Duke University (email: bb1@duke.edu). We would like to thank Jinzhao Du, Oded Koenigsberg, Woochoel Shin, Bo Zhou, and seminar participants in Peking University for their helpful comments. We are grateful to the associate editor and the anonymous referee for their constructive suggestions. We acknowledge the financial support from the National Natural Science Foundation of China (Grant No. 71703017) and Beijing National Natural Science Foundation (Grant No. 9184031). 


\section{Introduction}

Stores will be pulling out the stops on deep discounts aimed at drawing customers into stores. But retail-industry veterans acknowledge that, in many cases, those bargains will be a carefully engineered illusion.

- Susanne Kapner, "The Dirty Secret of Black Friday 'Discounts'”, Wall Street Journal, November 25,2013

Retail price promotions purport to provide extra value to consumers and these promotions often result in large increases in sales. However, not all retail price promotions provide consumers with additional monetary value. For instance, a retailer might raise the retail price of an item just before a sale and then "promote" the product by listing the initial price as the sales price. Such a practice was alluded to in above referenced Wall Street Journal article entitled "The Dirty Secret of Black Friday 'Discounts' " which states that retail discounts generally are not discounts at all - they are priced into it from the beginning. The article notes that "big retailers work backward with their suppliers to set starting prices that, after all the markdowns, will yield the profit margins they want". The net result is that between 2009 and 2012, the number of deals offered by 31 major department stores and apparel retailers has increased $63 \%$ and the average discount jumped to $36 \%$ from $25 \%$, but the gross margins of the same retailers have remained flat at $27.9 \%$.

The Wall Street Journal is not the only one to note such practices, and such practices are also observed for online retailers. For example, in December 2014, two consumers filed a class action lawsuit against online retailer Amazon.com, alleging the company's practice of stating a product's "listed price" as the price of some high priced, low volume competitor to mislead customers into believing they are saving money. ${ }^{1}$ Specifically, the lawsuit claims that Amazon "cherry-picks the highest price it can find for the item and uses it to create a significant price discrepancy and the impression of considerable savings for its customers... The reality is that the Amazon price is no different than the price of competitors, and no discount is provided over Amazon.com's everyday pricing." As the result, the savings are "illusory and/or grossly overstated".

Such practices of trying to set a higher reference price are also observed for store brands, where retailers have more control over the list price. For example, in July 2015, two consumers

\footnotetext{
${ }^{1}$ https://www.truthinadvertising.org/wp-content/uploads/2015/02/Fagerstrom-v-Amazon-notice-ofremoval-and-complaint.pdf
} 
in California filed a federal class action suit alleging that discount department store Kohl's offered phony discounts on items they bought — a Jennifer Lopez dress, a Sonoma robe and an Apt. 9 shirt - from three of Kohl's private-label or exclusive lines. ${ }^{2}$ Their lawyers wrote in their initial complaint that "As a result... Plaintiffs and members of the proposed Class... received items of lesser value and quality than they expected and Kohl's unlawfully, inequitable, and otherwise improperly was thereby unjustly enriched and benefited."

We label such practices as "false promotions" in that these price-promoting tactics make the promoted product seem more appealing to consumers than they would be, should they know the complete pricing history and/or available pricing options. A false promotion is formally defined in this paper as an advertised discount off a high price that never or rarely actually is charged by either the promoting store or some other referenced store. Correspondingly, there are two general types of false promotions, within-store false promotions and between-store false promotions. Within-store false promotions occur if the advertised regular price has never been the actual selling price in the same store; the Black Friday scenario falls into this category. Between-store false promotions occur when the regular price referred to in one store is consistently inflated to a level that has never been charged by that store or the vast majority of competitors. The Amazon scenario falls into this second category. Our game theoretic model results in equilibria that reflect both cases.

We acknowledge that false promotions as defined by us may not be viewed as illegal. The Federal Trade Commission (FTC)'s "Guidelines Against Deceptive Pricing” states that products should be sold at their regular prices before going on sale for a "reasonably substantial period of time". Likewise, the California Supreme Court ruled that retailers' misrepresentations about the price of goods are actionable if a consumer suffers economic harm when this consumer "purchased a product that he or she paid more for than he or she otherwise might have been willing to pay if the product had been labeled accurately." However, the FTC guidelines provide no definition of what constitutes a "reasonably substantial period of time", and the California Supreme Court leaves open the question of whether or not the consumers are deceived by such false promotions. Regarding the class action lawsuit against Amazon that is described earlier, Amazon says in its motion to dismiss the class action lawsuit that in the process of making their purchases, the plaintiffs expressly accepted Amazon's Conditions of Use (COUs) and the arbitration agreement within the

\footnotetext{
${ }^{2}$ https://consumermediallc.files.wordpress.com/2015/07/3-15-cv-01624-jah-wvg.pdf
} 
COUs. Amazon has also cited its arbitration agreements in other class action lawsuits and courts have upheld those agreements. ${ }^{3}$ When online retailers use such COUs to protect their actions, the legality of these pricing practice becomes unclear. Amazon also has a disclosure on its website stating the list price can have many origins: It can be the price on the product itself, it can be the price suggested by the manufacturer or supplier, or it can be Amazon's guess as to what the list price should be, which "may or may not" represent the prevailing price "in every area on any particular day" (Streitfeld, 2016).

Given this uncertainty about the legality of what we define as "false promotions", there are important theoretical questions as to why and when such practices might exist. The purpose of our study is to investigate how false promotions affect consumer choices and in turn retailers' optimal strategy, and in the process contribute to both the theoretical and behavioral IO literature. Specifically, we address the following questions: First, assuming at least some consumers' attitudes towards false promotions are negative, could a firm still benefit from giving such false promotions? Second, in what conditions is the firm more likely to give a false promotion? Finally, what are the profit implications for the industry at large?

To address these questions, we construct an analytical model consisting of two segments of consumers and two competing retail firms. More specifically, we let the duopoly retail firms sell substitutable products in a finite number of periods to consumers. They can choose to sell their products at a regular price exogenously set by the manufacturer, offer real promotions, or offer false promotions. In the latter two cases the retail firms set the optimal discount rate as well as the type of promotion. The firms' choice of promotion strategies affects consumer knowledge on the existence of false promotions, which in turn affects consumer word-of-mouth (WOM) and subsequent choices. Both firms are forward-looking and maximize their discounted profit over a finite horizon. In each period, the consumers observe the claimed promotional offering, and then make purchase decisions to maximize their one-period utility. One segment of consumers is sophisticated and is able to discern false promotions. The other segment is naive and trusts the claimed promotional offering. Both segments of consumers are located evenly on a Hotelling line. If false promotions are observed in the first period, the existence of false promotions within the

\footnotetext{
${ }^{3}$ http://topclassactions.com/lawsuit-settlements/lawsuit-news/50566-amazon-appeals-arbitrationlist-price-class-action-dismissal/
} 
industry is known, and sophisticated consumers pass this information via WOM to a fraction of naive consumers, who then become sophisticated in the next period. Therefore, WOM serves as a monitoring mechanism to incentivize the firms to behave honestly.

We find surprisingly, that the possibility of offering false promotions can improve the payoffs for both firms in situations of high competition. This is because when the option of offering a false promotion is not available, a real promotion is always the best response when the other firm provides a real promotion; but when false promotions become an option, this is not necessarily the case. We also find that even when some consumers can identify false promotions and pass on their knowledge of this practice to others, a firm may still find it optimal to offer such promotions from time to time. In general, a firm is more likely to offer false promotions when competition is less fierce, when the size of the sophisticated segment is smaller, and when the effect of WOM is weaker. We also find that in highly competitive situations, false promotions occur almost as often in periods where the negative implications of giving a false promotion carry over to future periods compared to the end period where there are no long-term effects. Our model also offers explanations to why we observe both the within-store and between-store false promotions. Although these results flow from our specified setting of raising the regular price and then offering a promotion, they should extend to other retailer tactics associated with consumer deception described by Lindsey-Mullikin and Petty (2011).

The remainder of the paper is organized as follows. First, $\S 2$ reviews the relevant literature. $\S 3$ then describes the model setting and $\S 4$ details the model solutions. Further, $\S 5$ discusses implications with alternative utility specifications, scenarios without WOM, and an equilibrium selection mechanism. Finally, $\S 6$ concludes with a schedule of next steps.

\section{Relevant Literature}

Our paper builds upon several streams of existing literature. The first concerns utility formulations based on reference-dependent preferences. Since the seminal work of Kahneman and Tversky (1979), reference-dependence has been an active domain of research in economics (e.g., Heidhues and Kőszegi, 2014; Kőszegi and Rabin, 2006; Lien and Zheng, 2015; Tversky and Kahneman, 1991; Zhang and Zheng, 2017) and numerous other fields such as marketing (e.g., Hardie et al., 1993) and psychology (e.g., Breiter et al., 2001; Weber et al., 2007). The basic assumption under reference- 
dependence is that people evaluate outcomes relative to a reference point by framing the evaluation decision in terms of gains and losses relative to that point. Such an assumption is compatible with fMRI data indicating buyers activate the reward processing orbitofrontal cortex during the buying process if there exists a salient reference price (Weber et al., 2007). This assumption also implies that firms can take advantage of this consumer tendency when designing pricing strategies. For example, Heidhues and Köszegi (2014) show that for any degree of consumer loss aversion, a monopolist's optimal price distribution consists of low and variable "sale" prices and a high and atomic "regular" price. Another related behavioral economics concept comes from Thaler's transaction utility theory (Thaler, 1985). This theory distinguishes between two types of utility, acquisition and transaction utility. Acquisition utility represents the economic gain from the purchased item and is measured by the utility of the good, net the price paid, whereas transaction utility denotes the psychological satisfaction and pleasure from the purchase and is measured by the internal reference price net the price paid. Firms often employ various tactics to manipulate the internal reference price and in turn the transaction utility associated with the product.

We build on these two behavioral concepts, reference-dependence and transaction utility, to specify consumers' utility from purchasing an item when it is on sale. Our conception is both similar and different from this work. It is similar in that it acknowledges that consumers get satisfaction (utility) when the observed price is lower than the reference price. However, it also departs from previous work in both the research objectives and the mechanism through which pricing strategies affect consumer choice. More specifically, we 1) focus on the optimal promotional tactics (real or false promotions) under competition in contrast to the optimal discount (which we treat as an endogenous decision), 2) account for the negative impact of identified false promotions on consumer utility instead of the negative reference gap, and 3) augment the setting by accounting for consumer heterogeneity and the impact of WOM.

A second related literature revolves around the empirical fact that short-term sales volumes increase substantially above what would be expected by the price reduction, i.e., there seems to be a bump in sales due entirely to the fact that the item is "on sale". For example, "price promotions" have been shown to lead to increased sales even when there is no price reductions (Inman and McAlister, 1993). Other studies demonstrate that after controlling for the price discount, "being on promotion" (i.e., the presence of displays and feature ads) still significantly increase brand choice 
(e.g., Chintagunta, 1992). These findings imply that even in the absence of a price reduction, the promotion signal alone can affect some consumers' value and thus alter choice.

With these empirical observations noted, most existing studies on the effects of price promotions are built upon an implicit assumption that the promotion is real, i.e., it results in an actual price reduction. Few, if any, studies have examined what might occur if the promotions claimed by retailers are false, both in terms of consumer response and retailer behavior. Perhaps the closest was conducted by Anderson and Simester (1998), who recognize that 1) if consumers value "promotions," stores may want to place sale signs on non-discounted products and 2) such decisions are endogenous. In this sense, sale signs might be "false". In addition, they demonstrate that when consumers cannot recognize initial and discounted price levels and consumers' price expectations are sensitive to the number of products that have sale signs, such false sale signs reduce demand for other products that already have sale signs. Anderson and Simester (2001) further show empirically that sale signs are less effective at increasing demand when more items have them. Therefore, customers implicitly regulate stores' temptation to place sale signs on every item by ascribing less credibility to sale signs when they see them on more products.

Similar to Anderson and Simester (1998)'s assumption that consumers lack knowledge on initial prices and respond positively to signs (i.e., purported discounts), we assume this is true for a fraction of consumers denoted as naive consumers. However, our work differs from Anderson and Simester (1998) in the mechanism of how sale signs work. In their work, sale signs may increase demand by providing consumers with credible information about which products are discounted. Instead our model draws upon prior reference-dependence research on the effects of perceived savings. Thus, in our case sale signs provide direct utility to consumers by changing their valuation of the products: a promotion perceived to be real increases consumer valuation, while a promotion perceived to be false decreases consumer valuation.

Finally, our study is also related to the literature on WOM learning. In this literature agents use information about the experiences of other agents to guide their own decisions (e.g., Banerjee and Fudenberg (2004); Cabral and Hortaçsu (2010); Ellison and Fudenberg (1995); Liu (2011); Rob and Fishman (2005); Board and Meyer-ter-Vehn (2013)). Probably the most relevant of these studies is Liu (2011), who models a single long-lived firm interacting with a sequence of short-lived consumers where reputation accumulates over time and is passed between generations of consumers. 
His interest however is quite different, in that he explores how this forward-looking opportunistic firm acts strategically to "fool" informationally constrained consumers in order to exploit them. Further, we abstract away the process of consumer information acquisition which he models as being determined endogenously, and assume instead that the firm's reputation is passed on via WOM cost-free. This simplifies our analysis to a large degree, yet we believe is not unrealistic given that retailers nowadays are often reviewed by consumers, and such reviews serve as the accumulated reputation for the retailers and are easy for consumers to acquire.

\section{Model Setup}

Our goal is to better understand if one or more firms might offer false promotions and if so, under what conditions would we expect these false promotions to be offered. This led us to construct a parsimonious five-parameter model with the following elements: The industry is composed of two retail firms offering similar, albeit not identical products, which are also separated spatially. This spatial separation provides them with some monopoly power. We capture the degree of separation with a parameter $d$. The products being sold are non-durable, consumed readily, and often. Firms simultaneously choose their promotion strategy (real, false, or no promotion) and the optimal discount off the stated list price. This stated list price is denoted $p^{\star}$ if the promotion is real and is higher than $p^{\star}$ if the promotion is false. Consumers are one of two types. The first are sophisticated and thus able to discern false promotions, the second are naïve and thus accept all promotions as being real. The proportion of sophisticated consumers is captured with the parameter $\lambda$. Consumers like promotions but dislike false promotions. Consumers have heterogeneous preferences for shopping at the two retail stores. They choose to shop at the retail store that maximizes their utility, which is a function of travel distance, the intrinsic value of the product (captured by the parameter $v$ ), the transaction price and the type of promotion being offered. Sophisticated consumers inform a subset of naïve consumers about the existence of false promotions if at least one firm gives a false promotion. And this subset of naïve consumers then becomes sophisticated in the next period. The efficiency of this WOM is captured by the parameter $\epsilon$.

Below we describe in details the competitive environment, consumers and WOM. 


\subsection{Competitive Environment}

In order to capture the competitive effects, our setting includes two retail stores: stores 1 and 2. Both stores live two periods and have a common discount factor of $\delta<1$. For simplicity, we assume this factor is close enough to 1. Each store sells one product with zero marginal cost. Both stores have a regular unit list price $p^{\star}$, which is set by the manufacturer (i.e., MSRP) and is assumed to be exogenous.

Store $i(i \in\{1,2\})$ in any given period can adopt one of three promotion strategies: offer 1$)$ no promotion and charge the list price $p^{\star} ; 2$ ) a real promotion that lists a regular price $p^{\star}$ and a discounted price $\left(1-\alpha_{i}\right) p^{\star}$, where $\alpha_{i}$ is the percent discount offered by store $i$; and 3$)$ a false

promotion that lists a regular price $\frac{p^{\star}}{1-\alpha_{i}}$, a discounted price $p^{\star}$ and claiming a percent discount of $\alpha_{i}$. By definition $0<\alpha_{i}<1$, and is set by store $i$. Thus the selling price is endogenous and the discount rate (or equivalently the percent off) is assumed to be the same across the two types of promotions, all else equal.

Denote the three promotion strategies by $N, T$, and $F$ respectively. Each store has a strategy space $\{\alpha,\{N, T, F\}\}$. We refer to an element in $\{N, T, F\}$ as the promotion strategy and $\alpha$ as the pricing strategy. Price competition is modeled with a Hotelling environment where the two stores are located at the two ends of a Hotelling line. For mathematical convenience we assume the length of the line is $\left(p^{\star}+v\right) d$, where $v$ is the consumers' valuation on the products. $d$ is a parameter that captures degree of competition: as $d$ increases, competition becomes less fierce. We place constraints on these three competitive environment parameters, $d, p^{\star}$ and $v$. Specifically we assume $d \in\left(\frac{1}{3}, 1\right)$ to ensure positive payoffs for both stores. Additionally, we assume $\frac{v}{p^{\star}} \in\left[\frac{4+2 d}{3-d}, \frac{2+d}{d}\right]$. The lower bound is needed to ensure that the intrinsic value of the product is high enough for all consumers to get positive utility from at least one product offering, i.e., the market is fully covered at the exogenously determined price, $p^{\star}$, while the upper limit is needed to ensure that the market shrinks if both stores adopt false promotions.

\subsection{Consumers}

There is a continuum of mass 1 consumers. Among all the consumers, a fraction of $\lambda$ are sophisticated and the rest are naive. Both types of consumers reside evenly on the Hotelling line. To purchase the product, consumers need to travel to a store. We assume the disutility from travelling 
equals the travel distance. Naive consumers are assumed to know nothing and when making decisions, they trust the claimed offering of each store as being truthful. Sophisticated consumers, on the other hand, know the strategy space is identical to both stores, but they do not know whether or not $F$ is included in the strategy space. Although their prior belief on $F$ being in the strategy space is $\mu$, once $F$ is used, sophisticated consumers identify the promotion is false and learn about the exact structure of the strategy space available to the industry, i.e, $\mu$ is updated to $\mu^{\prime}=1$. The net result of this update is that sophisticated consumers begin to spread WOM and educate a subset of naive consumers who become sophisticated in the next period. In this way $\mu^{\prime}=1$ is equivalent to saying that the industry has used at least one false promotion.

In each period, a consumer maximizes her one-period utility ${ }^{4}$ by choosing whether to purchase and where to purchase. We choose a functional form for this utility function that captures the intrinsic value of the product, the cost of the product, the disutility of having to travel to purchase the product, and the added utility (disutility) of purchasing a product on a real (false) promotion. This latter component of utility can be thought of as coming from a reference-dependent formulation where the transaction price is lower than the listed reference price, or more straightforwardly, a boost or decrement associated with the transaction utility from Thaler's transaction utility theory. Specifically, we assume the utility from purchasing the product under no promotion is $u(N)=$ $v-p^{\star}-x$, where $v$ is the utility of consumption and $x$ is the travel distance. For consumers who think they purchase the product through a real promotion, we assume the following utility formulation: $u(T)=(1+\alpha) v-p-x$. Here, $p \in\left\{p^{\star},(1-\alpha) p^{\star}\right\}$ is the price offered by the store which they purchase from. Thus, $p=p^{\star}$ if the promotion is false and $p=(1-\alpha) p^{\star}$ if the promotion is real. Essentially, $v-p-x$ corresponds to the acquisition utility in Thaler's transaction utility theory whereas $\alpha v$ corresponds to the transaction utility. If consumers think the purchase is through a false promotion, the utility decreases to $u(F)=(1-\alpha) v-p^{\star}-x$, i.e., $\alpha v$ now captures the negative affect associated with knowing that the firm is engaged in "poor" marketing practices.

The utility function is summarized in Table 1 by firm strategy and consumer belief. Hence, for a sophisticated consumer, the utility from purchasing under strategy $T$ is the highest, followed by

\footnotetext{
${ }^{4}$ We assume consumers are myopic, which is similar to a few existing studies (e.g., Kovác̆ and Schmidt, 2014; Liu, 2011; Taylor, 2004). Research in behavioral economics shows that consumers may have bounded rationality and may not know or care about future prices (Ellison, 2006), and it is rather common in the industrial organization literature to model firms as perfectly rational while consumers use only simple decision rules (Kovác̆ and Schmidt, 2014).
} 
$N$, and then $F$. For the naive consumer the order is $T, F$ and then $N$. We later show that under this model setting, the parameters $p^{\star}$ and $v$ reduce to one parameter $\frac{v}{p^{\star}}$.

\begin{tabular}{|c|c|c|c|c|}
\hline \multirow{4}{*}{ Firm Strategy } & & \multicolumn{3}{|c|}{ Consumer Belief } \\
\hline & $N$ & $v-p^{\star}-x$ & - & - \\
\hline & $T$ & - & $(1+\alpha) v-(1-\alpha) p^{\star}-x$ & - \\
\hline & $F$ & - & $(1+\alpha) v-p^{\star}-x$ & $(1-\alpha) v-p^{\star}-x$ \\
\hline
\end{tabular}

\subsection{Word-of-Mouth}

WOM happens if and only if the sophisticated consumers know for sure the exact strategy space of the two stores. In this case, in the next period, $\epsilon$ of naive consumers become sophisticated through WOM, and can discern false promotions. The parameter $\epsilon \in[0,1-\lambda]$ indicates the effectiveness of WOM. WOM is fully effective if $\epsilon=1-\lambda$, meaning the entire population of the naive consumers (and hence the entire population) knows a promotion can be false. In contrast, WOM does not work if $\epsilon=0$, in which case the model reduces to a simple repeated game.

In summary, we define the market with two exogenous factors, $\frac{v}{p^{\star}}$ and $d$, and the consumer market with two exogenous factors, $\epsilon$ and $\lambda$. These factors are constrained and also interrelated. As alluded to before, these constraints are imposed to ensure the following: 1) The stores are competing for the marginal consumer (i.e., the market is fully covered) unless both stores offer $F$. This requires $\frac{v}{p^{\star}}>\frac{4+2 d}{3-d}$. In words, the value to price ratio is high enough for all consumers to buy a product. 2) The industry demand decreases when both stores offer $F$. Specifically, some sophisticated consumers may decide not to buy any product if they believe both stores are offering false promotions. This restriction bounds the value to price ratio on the high side, i.e., $\frac{v}{p^{\star}}<\frac{2+d}{d}$. (Note, however, that sophisticated consumers are not precluded from buying the product even though they know the product is being sold via a false promotion. This can occur if the store is close enough to overcome the negative impact of the false promotion.) These restrictions, along with our assumed utility functions, allow us to derive firm demand functions and equilibrium solutions for a broad range of parameter settings that characterize the consumer market, i.e., $\epsilon$ and $\lambda$, and the market place, i.e., $\frac{v}{p^{\star}}$ and $d$. We next solve for equilibrium conditions as a function of these exogenous parameters. 


\section{Model Analyses}

We start our model analysis by deriving the optimal pricing strategies and consumer demand in a single period (§4.1). Based on these results, we first derive the benchmark equilibria when false promotions are not allowed $(\S 4.2)$. We then add false promotions into the strategy space and derive the corresponding equilibria ( $\$ 4.3$ and $\S 4.4)$. Analytical solutions are presented whenever feasible, and numerical results are presented when analytical results are prohibitively complex to obtain. We conclude the section by summarizing all equilibria with additional numerical insights (§4.5).

\subsection{Optimal Strategy in One Period}

Because each store can take one of the three promotion strategies $T, F$ and $N$ in each period, there are 9 promotion strategy profiles. We consider the optimal pricing (discount rate) strategy under each promotion strategy profile. The optimal price under $T$ is solved as the profit-maximizing price given the other store's promotion strategy profile. The optimal price under $F$ is assumed to mimic that of $T$. For instance, when store 1 chooses $F$ and store 2 chooses $T$, store 1 sets the discount rate to be its discount rate when both stores choose $T, \alpha_{1}^{T T}$. Hence, store 1 inflates its regular price to $\frac{p^{\star}}{1-\alpha_{1}^{T T}}$. We do not assume store 2's optimal discount rate is $\alpha_{2}^{T T}$, but instead allow store 2 to react to store 1's pricing strategy. In this way we are conservative by making it harder for store 1 to select $F$. Also, note that when $F$ appears in the strategy profile, decisions of sophisticated consumers and naive consumers differ, and thus need to be considered separately. To conserve space, we derive the results for $\{N, N\},\{T, T\}$ and $\{F, F\}$ in the main paper and leave the rest in Appendix A.

$\{N, N\}$ : When the promotion strategy profile is $\{N, N\}$, both stores charge the regular price $p^{\star}$. Each consumer can be uniquely indexed by her distance to store 1 denoted as $x$. Consumer $x$ obtains utility $u_{1}(x)=v-p^{\star}-x$ when purchasing from store 1 and obtains utility $u_{2}(x)=$ $v-p^{\star}-\left[\left(v+p^{\star}\right) d-x\right]$ when purchasing from store 2. The marginal consumer, denoted $x^{N N}$, is indifferent between purchasing from the two stores, i.e., $u_{1}\left(x^{N N}\right)=u_{2}\left(x^{N N}\right)$, leading to $x^{N N}=\frac{\left(v+p^{\star}\right) d}{2}$. All consumers who are closer to store $1(2)$ than consumer $x^{N N}$ purchase from store 1 (2). This implies each store gets demand $\frac{\left(v+p^{\star}\right) d}{2}$ and obtains payoff $\frac{p^{\star}\left(v+p^{\star}\right) d}{2}$. We normalize all payoffs by this amount hereafter. The normalized payoff of both stores is therefore 1. Full market coverage requires $u_{i}(x)>0(i=1,2)$, i.e., $\frac{v}{p^{\star}}>\frac{2+d}{2-d}$, which is satisfied under our assumption. 
$\{T, T\}$ : When the promotion strategy profile is $\{T, T\}$, each store has a real promotion with discount rate $\alpha_{i}^{T T}, i=1,2$. Consumer $x$ obtains utility $u_{1}(x)=\left(1+\alpha_{1}^{T T}\right) v-\left(1-\alpha_{1}^{T T}\right) p^{\star}-x$ when purchasing from store 1 and obtains utility $u_{2}(x)=\left(1+\alpha_{2}^{T T}\right) v-\left(1-\alpha_{2}^{T T}\right) p^{\star}-\left[\left(v+p^{\star}\right) d-x\right]$ when purchasing from store 2 . The marginal consumer is located at $x^{T T}=\frac{\left(v+p^{\star}\right)\left(\alpha_{1}^{T T}-\alpha_{2}^{T T}+d\right)}{2}$. Hence, the normalized payoff of store 1 is $\frac{\left(1-\alpha_{1}^{T T}\right)\left(\alpha_{1}^{T T}-\alpha_{2}^{T T}+d\right)}{d}$, leading to store 1's optimal discount rate $\alpha_{1}^{T T}=\frac{1+\alpha_{2}^{T T}-d}{2}$. Store 2's optimal discount rate is $\alpha_{2}^{T T}=\frac{1+\alpha_{1}^{T T}-d}{2}$ by symmetry. Hence, the optimal discount rates are $\alpha_{1}^{T T}=\alpha_{2}^{T T}=1-d$, i.e., lower in a less competitive environment, and the demand is $\left(\frac{\left(v+p^{\star}\right) d}{2}, \frac{\left(v+p^{\star}\right) d}{2}\right)$. The normalized payoffs are $(d, d) . u_{i}>0(i=1,2)$ requires $\frac{v}{p^{\star}}>\frac{3 d}{4-3 d}$ and is satisfied under our assumption.

$\{F, F\}$ : When the promotion strategy profile is $\{F, F\}$, each store has a false promotion with discount rate $\alpha^{F F}=\alpha^{T T}=1-d$. Sophisticated consumer $x$ obtains utility $u_{s 1}(x)=\left(1-\alpha^{T T}\right) v-$ $p^{\star}-x$ when purchasing from store 1 and utility $u_{s 2}(x)=\left(1-\alpha^{T T}\right) v-p^{\star}-\left[\left(v+p^{\star}\right) d-x\right]$ when purchasing from store 2. The sophisticated consumer who is indifferent between purchasing from the two stores is located at $x_{s}^{F F}=\frac{\left(p^{\star}+v\right) d}{2}$. However, it is easy to show that this marginal sophisticated consumer gains strictly negative utility due to the restriction $\frac{v}{p^{\star}}<\frac{2+d}{d}$, so the market is not fully covered for these consumers. The sophisticated consumer $x_{s 1}^{F F}$ who is indifferent between not purchasing and purchasing from store 1 satisfies $\left(1-\alpha^{T T}\right) v-p^{\star}-x_{s 1}^{F F}=0$, so that $x_{s 1}^{F F}=d v-p^{\star}$. Similarly, the consumer who is indifferent between not purchasing and purchasing from store 2 is located at $x_{s 2}^{F F}=\left(v+p^{\star}\right) d-\left(d v-p^{\star}\right)$. Hence, for each store, the demand from sophisticated consumers is $d v-p^{\star}$. The demand from naive consumers is the same as under the promotion strategy profile $\{T, T\}, \frac{\left(v+p^{\star}\right) d}{2}$. Taken together, the total demand of each store is $\tilde{\lambda}\left(d v-p^{\star}\right)+(1-\tilde{\lambda}) \frac{\left(p^{\star}+v\right) d}{2}$, leading to a normalized payoff of $1-\tilde{\lambda}\left[\frac{(2+d) p-d v}{d(v+p)}\right]$. $\tilde{\lambda}$ is the population of sophisticated consumers. In period $1, \tilde{\lambda}=\lambda$. In period $2, \tilde{\lambda}=\lambda$ if no store offered false promotions in period 1 , and $\tilde{\lambda}=\lambda+\epsilon$ otherwise. Let $u_{n i}$ denote the utility of naive consumers who purchase from store $i . u_{n i}>0(i=1,2)$ requires $\frac{v}{p^{\star}}>\frac{2+d}{4-3 d}$ and is satisfied under our assumption.

Proposition 1 summarizes all 9 promotion strategy profiles with both stores' optimal pricing strategies and corresponding payoffs. 
Proposition 1. Given promotion strategies in one period, the optimal pricing strategies and consumer demand are as follows: ${ }^{5}$

\begin{tabular}{cccccc}
\hline $\begin{array}{c}\text { Promotion } \\
\text { Strategy }\end{array}$ & $\alpha_{1}$ & $\alpha_{2}$ & $x_{s}$ & $x_{n}$ & Normalized Payoffs \\
Profile & & & & & \\
\hline$\{N, N\}$ & 0 & 0 & $\frac{\left(p^{\star}+v\right) d}{2}$ & $\frac{\left(p^{\star}+v\right) d}{2}$ & $(1,1)$ \\
$\{N, T\}$ & 0 & $\frac{1-d}{2}$ & $\left(\frac{1+d}{4}\right)\left(p^{\star}+v\right)$ & $\left.\frac{1+d}{4}\right)\left(p^{\star}+v\right)$ & $\left(\frac{3 d-1}{2 d}, \frac{(1+d)^{2}}{4 d}\right)$ \\
$\{N, F\}$ & 0 & $\frac{1-d}{2}$ & $\frac{\left(p^{\star}+v\right) d+\alpha_{2} v}{2}$ & $\frac{\left(p^{\star}+v\right) d-\alpha_{2} v}{2}$ & $\left(1+\frac{(1-d)(2 \tilde{\lambda}-1) v}{2\left(v+p^{\star}\right) d}, 1-\frac{(1-d)(2 \tilde{\lambda}-1) v}{2\left(v+p^{\star}\right) d}\right)$ \\
$\{T, N\}$ & $\frac{1-d}{2}$ & 0 & $\left.\frac{(1+d}{4}\right)\left(p^{\star}+v\right)$ & $\frac{(1+d)\left(p^{\star}+v\right)}{4}$ & $\left(\frac{(1+d)^{2}}{4 d}, \frac{3 d-1}{2 d}\right)$ \\
$\{T, T\}$ & $1-d$ & $1-d$ & $\frac{\left(p^{\star}+v\right) d}{2}$ & $\frac{\left(p^{\star}+v\right) d}{2}$ & $(d, d)$ \\
$\{T, F\}$ & $\alpha^{\star}(\tilde{\lambda})$ & $1-d$ & $\frac{\left(p^{\star}+v\right)\left(d+\alpha_{1}\right)+\alpha_{2} v}{2}$ & $\frac{\left(p^{\star}+v\right)\left(d+\alpha_{1}\right)-\alpha_{2} v}{2}$ & $\left(\pi_{1}(\tilde{\lambda}), \pi_{2}(\tilde{\lambda})\right)$ \\
$\{F, N\}$ & $\frac{1-d}{2}$ & 0 & $\frac{\left(p^{\star}+v\right) d+\alpha_{2} v}{2}$ & $\frac{\left(p^{\star}+v\right) d-\alpha_{2} v}{2}$ & $\left(1-\frac{(1-d)(2 \tilde{\lambda}-1) v}{2\left(v+p^{\star}\right) d}, 1+\frac{(1-d)(2 \tilde{\lambda}-1) v}{2\left(v+p^{\star}\right) d}\right)$ \\
$\{F, T\}$ & $(1-d)$ & $\alpha^{\star}(\tilde{\lambda})$ & $\frac{\left(p^{\star}+v\right)\left(d+\alpha_{1}\right)-\alpha_{2} v}{2}$ & $\frac{\left(p^{\star}+v\right)\left(d+\alpha_{1}\right)+\alpha_{2} v}{2}$ & $\left(\pi_{2}(\tilde{\lambda}), \pi_{1}(\tilde{\lambda})\right)$ \\
$\{F, F\}$ & $1-d$ & $1-d$ & $d v-p^{\star}$ & $\frac{\left(p^{\star}+v\right) d}{2}$ & $\left(\pi^{F F}(\tilde{\lambda}), \pi^{F F}(\tilde{\lambda})\right)$ \\
\hline where $\alpha_{1}$ and $\alpha_{2}$ are respectively the optimal discount rate of store 1 and store 2 and is lower
\end{tabular}
in a less competitive environment. For all cases except $\{F, F\}, x_{s}\left(x_{n}\right)$ is the location of a sophisticated (naive) consumer who is indifferent between purchasing from the two stores. For $\{F, F\}$, $x_{s}$ represents the location of a sophisticated consumer who is indifferent between purchasing from store 1 and not purchasing. $x_{n}$ represents the location of a naive consumer who is indifferent between purchasing from the two stores. $\tilde{\lambda}$ is the population of sophisticated consumers. In period 1 , $\tilde{\lambda}=\lambda$. In period 2, $\tilde{\lambda}=\lambda$ if no store offered false promotions in period 1, and $\tilde{\lambda}=\lambda+\epsilon$ otherwise. $\alpha^{\star}(\tilde{\lambda})=(1-d)\left(\frac{p^{\star}+2(1-\tilde{\lambda}) v}{2\left(p^{\star}+v\right)}\right) \cdot \pi^{F F}(\tilde{\lambda})=1-\tilde{\lambda}\left[\frac{(2+d) p-d v}{d(v+p)}\right]$ is the profit of each firm when both offer $F . \pi_{1}(\tilde{\lambda})=\frac{\left(1-\alpha_{1}^{\star}(\tilde{\lambda})\right)^{2}}{d}$ is the profit of the firm offering $T$ when the other firm offers $F$, and $\pi_{2}(\tilde{\lambda})=2-\frac{1-\alpha_{1}^{\star}(\tilde{\lambda})}{d}$ is the profit of the firm offering $F$ when the other firm offers $T$.

\subsection{Equilibria When False Promotions Are Not Allowed}

One of our goals is to see how the possibility of offering false promotions affects industry profit. Therefore, as a benchmark, we solve the model when $F$ is not in the strategy space.

\footnotetext{
${ }^{5}$ All payoffs are normalized by a factor of $\frac{p^{\star}\left(p^{\star}+v\right) d}{2}$.
} 
The game played by the two stores is a two-period repeated game with the following payoff matrix for the stage game:

Store 2

\begin{tabular}{cc|c|c|} 
& \multicolumn{2}{c}{$N$} & \multicolumn{1}{c}{$T$} \\
\cline { 3 - 4 } Store 1 & $N$ & 1,1 & $\frac{3 d-1}{2 d}, \frac{(1+d)^{2}}{4 d}$ \\
\cline { 3 - 4 } & \multirow{2nyy}{*}{} & $\frac{(1+d)^{2}}{4 d}, \frac{3 d-1}{2 d}$ & $d, d$ \\
\cline { 3 - 4 } & & &
\end{tabular}

When $d<0.5$, this stage game has a unique Nash equilibrium where both stores choose T. Therefore, the repeated game has a unique subgame perfect Nash equilibrium (SPNE) $\{\{T, T\},\{T, T\}\}$. The two-period equilibrium payoff of each store is then $d+\delta d$. In this SPNE, the payoff of each store is strictly lower than under $\{\{N, N\},\{N, N\}\}$. In other words, when competition is high, the firms always promote and get lower profits than if they never promote.

When $d>0.5$, this stage game has two Nash equilibria: $\{T, N\}$ and $\{N, T\}$. Hence, there are 4 pure-strategy SPNEs: $\{\{T, N\},\{T, N\}\},\{\{T, N\},\{N, T\}\},\{\{N, T\},\{T, N\}\}$, and $\{\{N, T\},\{N, T\}\}$. Now we see a reduction in promotion activity, but it is still at a significant level.

Note that $\{N, N\}$ cannot appear in any SPNE, because in both periods, $N$ is always dominated by $T$ if the other store's strategy is $N$.

Below, we discuss the case where $F$ is another promotion strategy. We first discuss the case where $d \in\left(\frac{1}{3}, \frac{1}{2}\right)$ with closed-form solutions $(\S 4.3)$. We then discuss the case where $d \in\left[\frac{1}{2}, 1\right)$ based on numerical solutions $(\S 4.4)$. $§ 4.5$ uses numerical simulations to summarize all cases with additional insights.

\subsection{Equilibria When False Promotions Are Allowed $(d<0.5)$}

As before the two stores play a dynamic game, which typically involve multiple equilibria. For parsimony we only consider pure-strategy equilibria. We find the additional option of offering a false promotion affects the equilibria substantially. The main result is that this possibility can improve the payoffs of both stores. Intuitively, as shown above, when $F$ is not feasible, choosing $T$ is the best response given the other store chooses $T$, hence in equilibrium, the two stores face the prisoner's dilemma in both periods. When $F$ becomes an additional strategy, $T$ is dominated by $F$ in some regions and hence may no longer be the equilibrium strategy for both stores. This helps the stores avoid the prisoner's dilemma in equilibrium. 
We demonstrate the existence of false promotions in equilibrium by solving the dynamic game over a wide range of parameter settings. As might be expected, the derived equilibria vary with parameter values and thus we look for a way of displaying these results in a parsimonious fashion. We start by noting that in order to determine the equilibrium solutions, we will need to determine the rank order of all the normalized payoffs given in Proposition 1. Moreover, the payoffs associated with all the promotion strategies involving one or more $F$ strategies are functions of the fraction of sophisticated consumers. This leads us to define two cutoff values for $\tilde{\lambda}$ that help determine the ranking between four of these payoffs, $d, \pi_{1}(\tilde{\lambda}), \pi_{2}(\tilde{\lambda})$ and $\pi^{F F}(\tilde{\lambda})$ and thus the existence of specific equilibria. We do this by defining two values of $\tilde{\lambda}$, denoted $\bar{\theta}$ and $\underline{\theta}$, where $\pi_{2}(\bar{\theta})=d$, and $\pi_{1}(\underline{\theta})=\pi^{F F}(\underline{\theta})$.

$\pi_{2}(\bar{\theta})=d$ implies:

$$
\bar{\theta}=\frac{2\left(p^{\star}+v\right) d-p^{\star}}{2 v}
$$

which is a function of the market parameters, $\frac{v}{p^{\star}}$ and $d$.

$\underline{\theta}$ can be characterized by the following lemma:

Lemma 2. $\underline{\theta} \in\left(\underline{\theta}_{\star}, \underline{\theta}^{\star}\right) \subseteq\left(0, \frac{1}{2}\right)$, where

$$
\underline{\theta}^{\star}=\frac{2\left(p^{\star}+v\right)\left(\frac{\sqrt{d}-d}{1-d}\right)-p^{\star}}{2 v},
$$

and $\underline{\theta}_{\star}$ satisfies

$$
\pi_{1}\left(\underline{\theta}_{\star} \mid \frac{v}{p^{\star}}=\frac{4+2 d}{3-d}\right)=\pi^{F F}\left(\underline{\theta}_{\star} \mid \frac{v}{p^{\star}}=\frac{4+2 d}{3-d}\right) .
$$

Proof. (See Appendix A)

We note that $\underline{\theta}$ can be larger or smaller than $\bar{\theta}$, as characterized in Proposition 3 .

Proposition 3. 1) There exists a $\frac{\hat{v}}{p^{\star}} \in\left(\frac{4+2 d}{3-d}, \frac{2+d}{d}\right)$, such that if $d \in\left(\frac{1}{3}, \frac{3-\sqrt{5}}{2}\right), \bar{\theta}>\underline{\theta}_{\star}$ and $\frac{v}{p^{\star}} \in\left(\frac{\hat{v}}{p^{\star}}, \frac{2+d}{d}\right)$, then $\bar{\theta} \leq \underline{\theta}$. 2) If $d \in\left(\frac{1}{3}, \frac{3-\sqrt{5}}{2}\right)$ and $\bar{\theta} \leq \underline{\theta}_{\star}$, then $\bar{\theta} \leq \underline{\theta}$. 3) In all other cases, $\bar{\theta}>\underline{\theta}$.

Proof. (See Appendix A) 
Proposition 3 provides the conditions that link the market characteristics $\frac{v}{p^{\star}}$ and $d$ with the ordering of $\underline{\theta}$ and $\bar{\theta}$ and thus the possible equilibria for any set of parameter values for $\lambda$ and $\epsilon$ (which determine $\tilde{\lambda}$ ), $\frac{v}{p^{\star}}$ and $d$. We use these results to search for pure-strategy SPNE using backward induction.

In the second period, the belief about the existence of false promotions, $\mu^{\prime}$, has two possibilities: $\mu^{\prime}=\mu$ if $F$ is not chosen in the first period and $\mu^{\prime}=1$ otherwise. Following Proposition 1 , the payoff matrix of the stage game in the second period is as follows:

Store 2

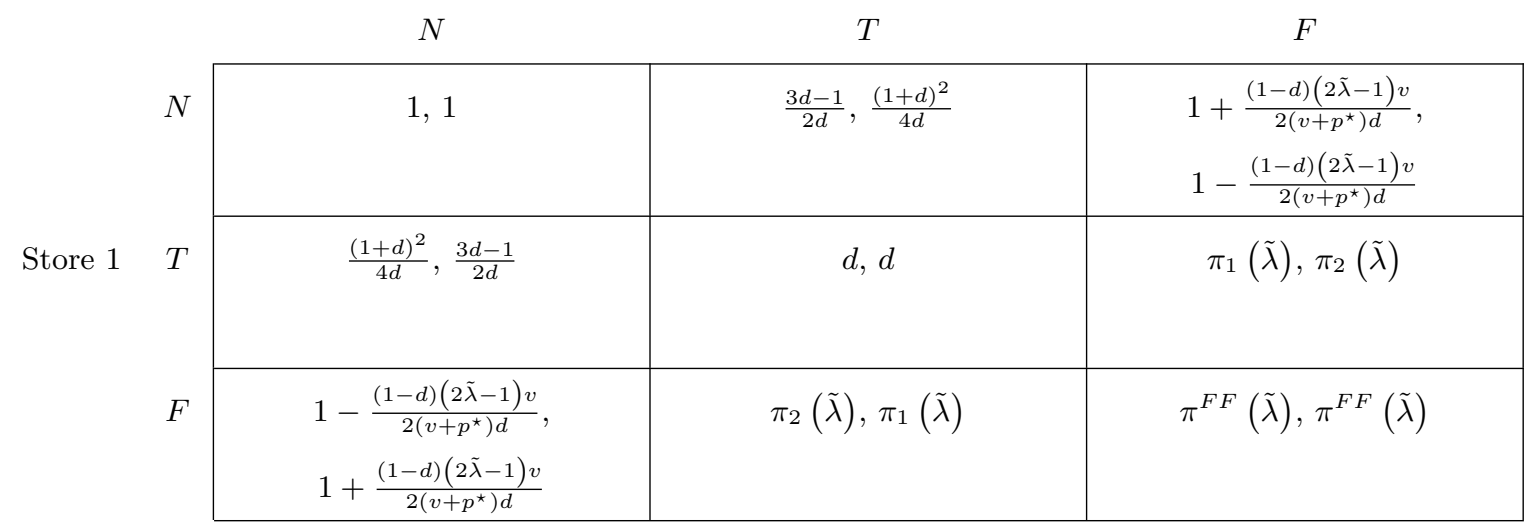

Based on this payoff matrix, we obtain the equilibria of this stage game under different market conditions as determined by the relationship between $\underline{\theta}, \bar{\theta}$ and $\tilde{\lambda}$ :

\begin{tabular}{cccc}
\hline Condition & $\tilde{\lambda}>\max \{\bar{\theta}, \underline{\theta}\}$ & $\min \{\bar{\theta}, \underline{\theta}\}<\tilde{\lambda}<\max \{\bar{\theta}, \underline{\theta}\}$ & $\tilde{\lambda}<\min \{\bar{\theta}, \underline{\theta}\}$ \\
\hline$\underline{\theta}<\bar{\theta}$ & $\{T, T\}$ & $\{T, F\},\{F, T\}$ & $\{F, F\}$ \\
$\underline{\theta}>\bar{\theta}$ & $\{T, T\}$ & $\{T, T\},\{F, F\}$ & $\{F, F\}$ \\
\hline
\end{tabular}

Several observations can be made from this table. First, depending on the relationship between $\underline{\theta}$ and $\bar{\theta}$, the equilibria differ across the two market conditions when the fraction of sophisticated consumers lies between the two cutoff values: when $\underline{\theta}<\bar{\theta}$, implying a larger $d$ (i.e., lower competition), the two stores adopt asymmetric strategies; when $\underline{\theta}>\bar{\theta}$, implying a smaller $d$ (i.e., higher competition), the two stores adopt symmetric strategies. As a result, the equilibria for the dynamic game will differ in corresponding regions. Second, regardless of the ordering of $\underline{\theta}$ and $\bar{\theta}$, when the fraction of sophisticated consumers is large, $\{T, T\}$ is the equilibrium, while when the fraction is small the equilibrium is $\{F, F\}$.

In the first period, the two-period payoffs of both stores depend on the fraction of sophisticated consumers $\lambda$ and the effectiveness of WOM $\epsilon$. When the fraction of sophisticated consumers and 
the effectiveness of WOM are both small enough (small $\lambda$ and $\epsilon$ ), there are two possible SPNEs as characterized by Proposition 4 .

Proposition 4. If $\lambda+\epsilon<\min \{\underline{\theta}, \bar{\theta}\}$, the subgame perfect Nash equilibria for the two period game are summarized in the following table:

\begin{tabular}{ccccc}
\hline SPNE & 1's Payoff & 2's Payoff & $\mu^{\prime}$ & Parameter Restriction \\
$\{\{F, F\},\{F, F\}\}$ & $\pi^{F F}(\lambda)+\delta \pi^{F F}(\lambda+\epsilon)$ & $\pi^{F F}(\lambda)+\delta \pi^{F F}(\lambda+\epsilon)$ & 1 & \\
$\{\{T, T\},\{F, F\}\}$ & $d+\delta \pi^{F F}(\lambda)$ & $d+\delta \pi^{F F}(\lambda)$ & $\mu$ & $\epsilon>\underline{\epsilon}_{1}$ \\
\hline
\end{tabular}

where $\underline{\epsilon}_{1}=\frac{\left[\alpha^{\star}(\lambda)-(1-d)^{2}\right]\left(p^{\star}+v\right)}{(2+d) p^{\star}-d v}, \pi^{F F}(x)$ is defined in Proposition 1.

In both SPNEs above, both stores have a payoff higher than the equilibrium payoff under the prisoner's dilemma.

Proof. In this case, the unique Nash equilibrium in the second period is $\{F, F\}$ for both $\mu^{\prime}=1$ and $\mu^{\prime}=\mu$. Hence, the forward-looking payoff matrix in the first period (i.e., the payoff matrix that takes into account future equilibrium outcomes) is as follows:

Store 2

\begin{tabular}{|c|c|c|c|c|}
\hline & & $N$ & $T$ & $F$ \\
\hline \multirow{3}{*}{ Store 1} & $N$ & $1+\delta \pi^{F F}(\lambda), 1+\delta \pi^{F F}(\lambda)$ & $\frac{3 d-1}{2 d}+\delta \pi^{F F}(\lambda), \frac{(1+d)^{2}}{4 d}+\delta \pi^{F F}(\lambda)$ & $\begin{array}{l}1+\frac{(1-d)(2 \lambda-1) v}{2\left(v+p^{\star}\right) d}+\delta \pi^{F F}(\lambda+\epsilon), \\
1-\frac{(1-d)(2 \lambda-1) v}{2\left(v+p^{\star}\right) d}+\delta \pi^{F F}(\lambda+\epsilon)\end{array}$ \\
\hline & $T$ & $\frac{(1+d)^{2}}{4 d}+\delta \pi^{F F}(\lambda), \frac{3 d-1}{2 d}+\delta \pi^{F F}(\lambda)$ & $d+\delta \pi^{F F}(\lambda), d+\delta \pi^{F F}(\lambda)$ & $\begin{array}{l}\pi_{1}(\lambda)+\delta \pi^{F F}(\lambda+\epsilon) \\
\pi_{2}(\lambda)+\delta \pi^{F F}(\lambda+\epsilon)\end{array}$ \\
\hline & $F$ & $\begin{array}{l}1-\frac{(1-d)(2 \lambda-1) v}{2\left(v+p^{\star}\right) d}+\delta \pi^{F F}(\lambda+\epsilon) \\
1+\frac{(1-d)(2 \lambda-1) v}{2\left(v+p^{\star}\right) d}+\delta \pi^{F F}(\lambda+\epsilon)\end{array}$ & $\begin{array}{l}\pi_{2}(\lambda)+\delta \pi^{F F}(\lambda+\epsilon) \\
\pi_{1}(\lambda)+\delta \pi^{F F}(\lambda+\epsilon)\end{array}$ & $\begin{array}{l}\pi^{F F}(\lambda)+\delta \pi^{F F}(\lambda+\epsilon), \\
\pi^{F F}(\lambda)+\delta \pi^{F F}(\lambda+\epsilon)\end{array}$ \\
\hline
\end{tabular}

There exists a Nash equilibrium $\{F, F\}$ in this game. Therefore, $\{\{F, F\},\{F, F\}\}$ is a SPNE. In addition, if $d+\delta \pi^{F F}(\lambda)>\pi_{2}(\lambda)+\delta \pi^{F F}(\lambda+\epsilon)$ or equivalently, $\epsilon>\underline{\epsilon}_{1},\{T, T\}$ is another Nash equilibrium in this game, so that $\{\{T, T\},\{F, F\}\}$ is another SPNE.

The intuition is as follows. Because the fraction of naive consumers is large enough and the effect of WOM is small, the profit from naive consumers is very large, which more than covers the lost sales to the (small number of) sophisticated consumers who would drop out of the market if both stores offer $F$. Therefore, in the second period, $F$ is the dominant strategy. In the first period, 
$F$ is the dominant strategy given the other store chooses $F$, because the existence of $F$ will lead to expansion of the aware segment anyway. Hence, $\{\{F, F\},\{F, F\}\}$ is a SPNE. However, when the effect of WOM exceeds a threshold, if the other store chooses $T$, then $T$ becomes the dominant strategy for the focal store since this strategy can maintain the current size of sophisticated segment in the next period. This gives rise to $\{\{T, T\},\{F, F\}\}$ as another SPNE.

Using a similar approach, we derive SPNEs under other parameter settings based on the relationship between $\underline{\theta}, \bar{\theta}$ and $\tilde{\lambda}{ }^{6}$ Table 2 summarizes the results. In all SPNEs, both stores enjoy payoffs that are higher than the equilibrium payoffs in the benchmark case where $F$ is not in the choice set.

\footnotetext{
${ }^{6}$ Details are provided in Appendix B.
} 
Table 2: SPNEs when $d<0.5$

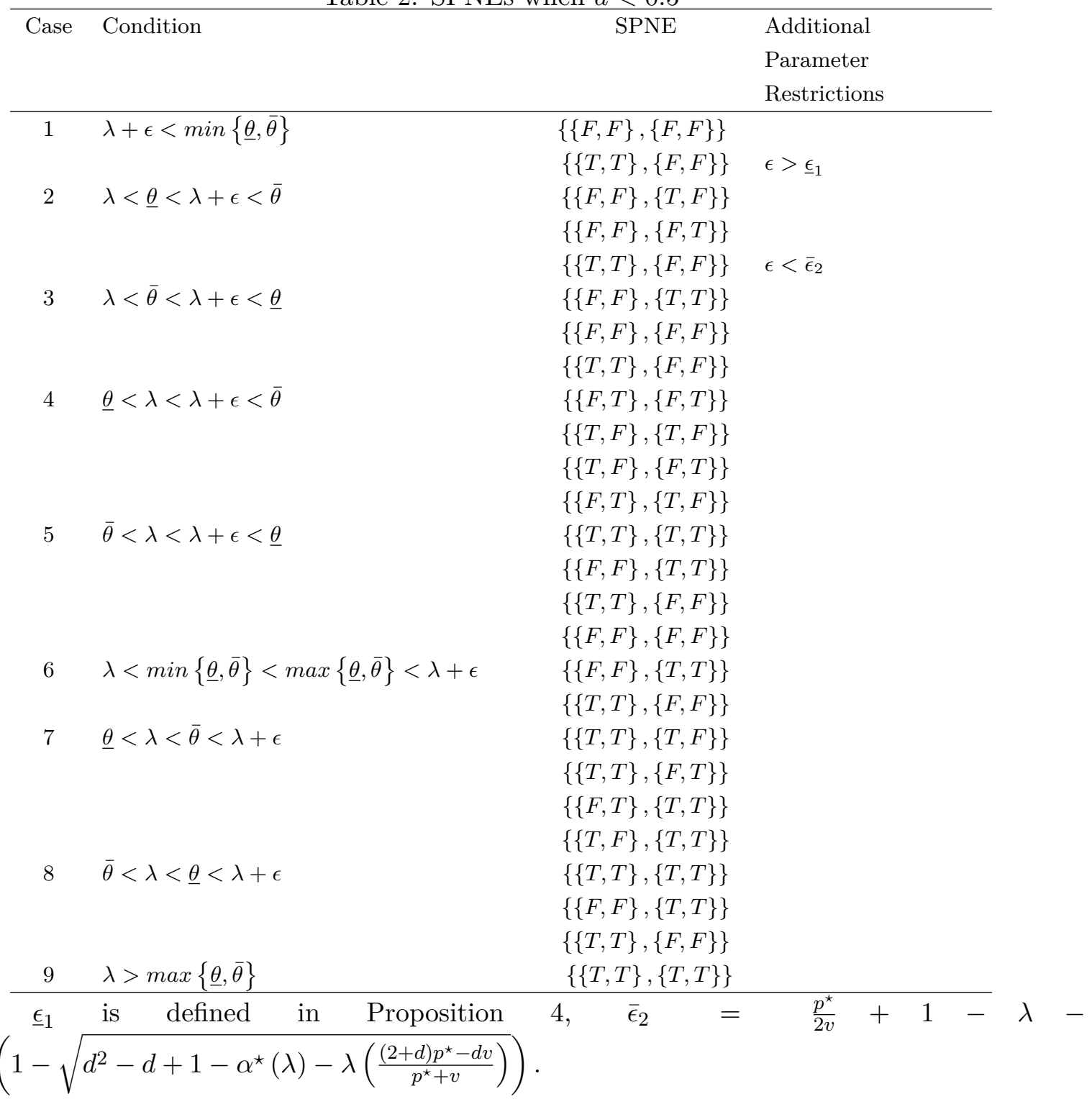

Figure 1 visualizes the above results by showing the equilibrium regions characterized by Case 1 - Case 9 in consumer space $(\lambda$ and $\epsilon)$, with the cutoffs reflecting the market place conditions $(\bar{\theta}$ and $\underline{\theta}$ ). Panel (a) corresponds to cases where $\underline{\theta}<\bar{\theta}$ (larger $d$ ) and Panel (b) corresponds to cases where $\underline{\theta}>\bar{\theta}$ (smaller $d)$. 


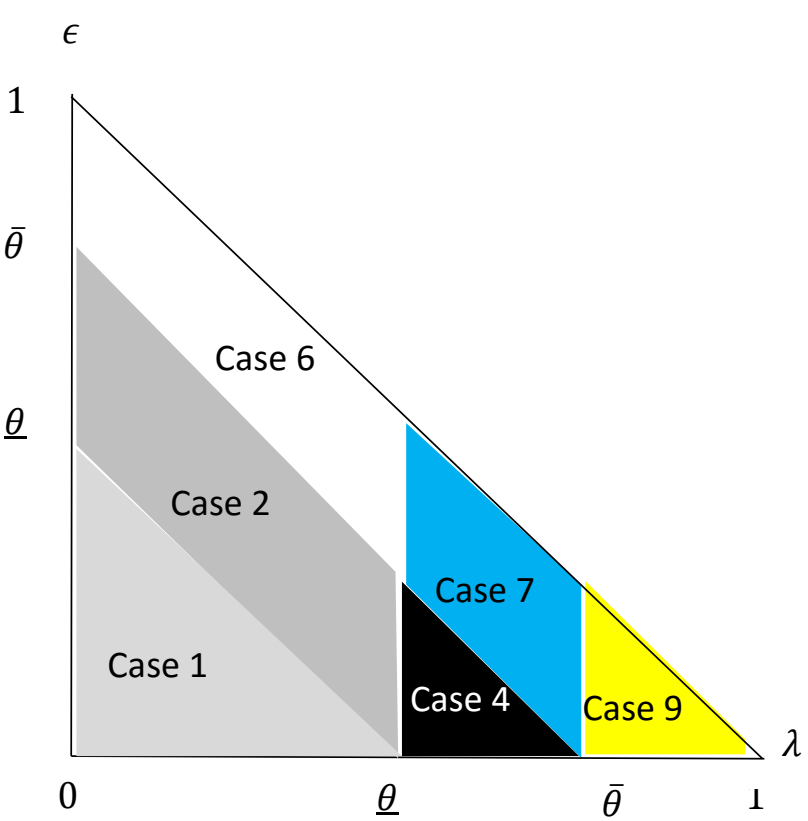

(a) $\underline{\theta}<\bar{\theta}$ $\epsilon$

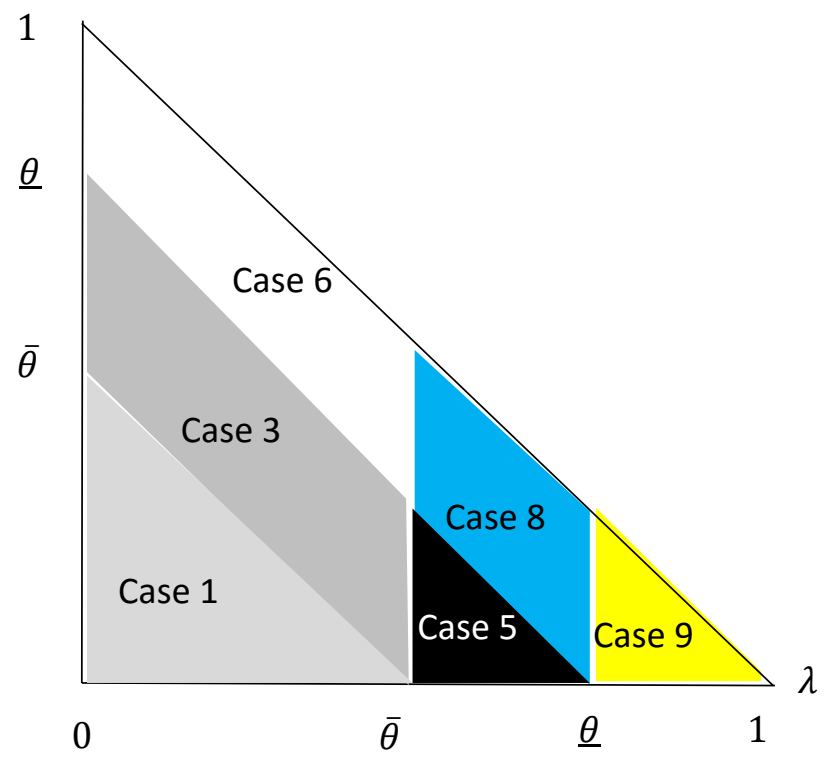

(b) $\underline{\theta}>\bar{\theta}$

Figure 1: Equilibrium Regions

These figures make clear that when the maximum fraction of sophisticated consumers $(\lambda+\epsilon)$ is small, there exists a SPNE where both stores choose $F$ in both periods. In contrast, when the initial fraction of sophisticated consumers $(\lambda)$ is large, the unique SPNE has both stores choosing $T$ in both periods. Under other parameter settings, we find a mix of real and false promotions in equilibria. In addition, we observe both within-store false promotions (i.e., the reference comes from own store in previous periods) and between-store false promotions (i.e., the reference comes from the other store) in equilibria. An example of within-store false promotions is equilibrium $\{\{F, F\},\{T, T\}\}$ characterized in Cases 3, 5,6 and 8. An example of between-store false promotions is equilibrium $\{\{F, T\},\{F, T\}\}$ characterized in Case 4.

When $\underline{\theta}<\bar{\theta}$, as $\bar{\theta}$ increases, the region supporting Case 9 shrinks, so the likelihood of observing a false promotion increases. More technically, because $\frac{\partial \bar{\theta}}{\partial d}>0$, the occurrence of false promotions increases with $d$. Hence, stores are more likely to offer false promotions when competition is less fierce. When $\lambda>\bar{\theta}$, false promotions are not observed, so the occurrence of false promotions decreases with the initial size of the sophisticated segment. When $\underline{\theta}>\bar{\theta}$, the relationships between different parameters and the occurrence of false promotions are less obvious. This led us to further explore the existence of false promotions via numerical analysis. We do this as follows. 
We take 50 values of $\lambda, \epsilon$, and $d$ that are evenly distributed in their respective support: $\lambda \sim$ $U(0,1), \epsilon \sim U(0,1)$, and $d \sim U\left(\frac{1}{3}, \frac{1}{2}\right)$. Conditional on $d$, we draw 50 values of $\frac{v}{p^{\star}}$ that satisfy $\frac{v}{p^{\star}} \in\left[\frac{4+2 d}{3-d}, \frac{2+d}{d}\right]$. This gives rise to $50^{4}$ sets of $\left[\lambda, \epsilon, d, \frac{v}{p^{\star}}\right]$. Because $\lambda+\epsilon$ cannot exceed $1,3,187,500$ sets of parameters are retained. Under each of these parameter sets, we check if the criteria for each equilibrium are satisfied, and in turn obtain all corresponding equilibria. Table 3 summarizes all possible equilibria and their occurrences. Overall, false promotions occur in $61.7 \%$ of all cases, reasonably evenly divided between first and second period. We find this interesting in that a firstperiod false promotion carries with it the implication that there are more sophisticated consumers in the second period and accordingly a larger impact of false promotions in the second period. In contrast, firms do not have to worry about future punishment due to WOM when making the promotion decision in the second period.

Table 3: Summary of SPNEs When $d<0.5$

\begin{tabular}{|c|c|c|}
\hline SPNE & Case & Occurrence \\
\hline \hline$\{\{F, F\},\{F, F\}\}$ & $1,3,5$ & $6.5 \%$ \\
\hline$\{\{F, F\},\{F, T\}\}$ & 2 & $6.5 \%$ \\
\hline$\{\{F, F\},\{T, F\}\}$ & 2 & $6.5 \%$ \\
\hline$\{\{F, F\},\{T, T\}\}$ & $3,5,6,8$ & $28.3 \%$ \\
\hline$\{\{F, T\},\{F, T\}\}$ & 4 & $3.0 \%$ \\
\hline$\{\{F, T\},\{T, F\}\}$ & 4 & $3.0 \%$ \\
\hline$\{\{F, T\},\{T, T\}\}$ & 7 & $17.5 \%$ \\
\hline$\{\{T, F\},\{F, T\}\}$ & 4 & $3.0 \%$ \\
\hline$\{\{T, F\},\{T, F\}\}$ & 4 & $3.0 \%$ \\
\hline$\{\{T, F\},\{T, T\}\}$ & 7 & $17.5 \%$ \\
\hline$\{\{T, T\},\{F, F\}\}$ & $1,3,5,6,8$ & $28.3 \%$ \\
\hline$\{\{T, T\},\{T, F\}\}$ & 7 & $17.5 \%$ \\
\hline$\{\{T, T\},\{F, T\}\}$ & 7 & $17.5 \%$ \\
\hline$\{\{T, T\},\{T, T\}\}$ & 5,9 & $38.3 \%$ \\
\hline
\end{tabular}

\subsection{Equilibria When False Promotions Are Allowed $(d \in[0.5,1))$}

When $d \geq 0.5$, analytical solutions are very difficult to obtain, so we solve for equilibria via numerical analysis. Similar to the previously discussed analysis, we take 50 values of $\lambda, \epsilon, d$ that are evenly distributed in their respective support: $\lambda \sim U(0,1), \epsilon \sim U(0,1)$, and $d \sim U(0.5,1)$. Conditional on $d$, we draw 50 values of $\frac{v}{p^{\star}}$ that satisfy $\frac{v}{p^{\star}} \in\left[\frac{4+2 d}{3-d}, \frac{2+d}{d}\right]$. Under each of the $3,187,500$ parameter settings where $\lambda+\epsilon \leq 1$, we solve the model and obtain all equilibria. Table 4 summarizes the eight cases with different equilibria. 
Table 4: SPNEs when $d \geq 0.5$

\begin{tabular}{cll}
\hline Case & SPNE & Occurrence \\
\hline 1 & $\{\{F, F\},\{F, F\}\}$ & $11.5 \%$ \\
2 & $\{\{F, F\},\{T, F\}\},\{\{F, F\},\{F, T\}\}$ & $10.2 \%$ \\
3 & $\{\{F, F\},\{N, T\}\},\{\{F, F\},\{T, N\}\}$ & $31.9 \%$ \\
4 & $\{\{T, F\},\{T, F\}\},\{\{T, F\},\{F, T\}\}$, & $3.8 \%$ \\
& $\{\{F, T\},\{T, F\}\},\{\{F, T\},\{F, T\}\}$ & \\
5 & $\{\{T, F\},\{N, T\}\},\{\{T, F\},\{T, N\}\}$, & $14.9 \%$ \\
& $\{\{F, T\},\{N, T\}\},\{\{F, T\},\{T, N\}\}$ & \\
6 & $\{\{N, T\},\{F, T\}\},\{\{T, N\},\{T, F\}\}, 2 \%$ \\
& $\{\{T, F\},\{N, T\}\},\{\{T, F\},\{T, N\}\}$, & \\
7 & $\{\{F, T\},\{N, T\}\},\{\{F, T\},\{T, N\}\}$ & \\
& $\{\{N, T\},\{N, T\}\},\{\{N, T\},\{T, N\}\}$, & \\
& $\{\{T, N\},\{N, T\}\},\{\{T, N\},\{T, N\}\}$, & \\
& $\{\{T, F\},\{N, T\}\},\{\{F, T\},\{T, N\}\}$ & \\
8 & $\{\{N, T\},\{N, T\}\},\{\{N, T\},\{T, N\}\}$, & \\
& $\{\{T, N\},\{N, T\}\},\{\{T, N\},\{T, N\}\}$, & \\
& $\{\{T, T\},\{N, T\}\},\{\{T, T\},\{T, N\}\}$, & \\
& $\{\{T, F\},\{N, T\}\},\{\{F, T\},\{T, N\}\}$ & \\
& &
\end{tabular}

Different from cases when $d<0.5$, we now find instances where a store does not promote (i.e., chooses $N$ ) and this occurs in both periods. In addition, we note that false promotions are always observed (in contrast, when $d<0.5,\{\{T, T\},\{T, T\}\}$ is the only equilibrium when $\lambda$ is large). As noted in $\S 4.2$, prisoner's dilemma does not take place if false promotions are not available. Consequently, the option of offering a false promotion does not necessarily lead to higher profits for both stores. For instance, if $\{\{T, N\},\{T, N\}\}$ is the equilibrium for the dynamic game without $F$, then when $F$ becomes an option, store 1 is worse off and store 2 is better off. Consequently total payoff for either store can be larger or smaller, depending on the new equilibrium.

Below we further explore the prevalence of false promotions and how the option to offer false promotions affects industry profits.

\subsection{Comparative Statics}

We pool the cases in $\S 4.3$ and $\S 4.4$ to further explore effects of different parameters on promotional outcomes and industry profits. ${ }^{7}$ We consider the percentages of $N, T$, and $F$ in each period, and the total profit of both stores in two periods. When there exist multiple equilibria, we take the average values across different equilibria as the expected value. For instance, in Case 6 in Table

\footnotetext{
${ }^{7}$ We take 50 values of $\lambda, \epsilon, d$ that are evenly distributed in their respective support: $\lambda \sim U(0,1), \epsilon \sim U(0,1)$, and $d \sim U\left(\frac{1}{3}, 1\right)$. Conditional on $d$, we draw 50 values of $\frac{v}{p^{\star}}$ that satisfy $\frac{v}{p^{\star}} \in\left[\frac{4+2 d}{3-d}, \frac{2+d}{d}\right]$.
} 
4, the expected numbers of $N, T$, and $F$ in period 1 are respectively $\frac{1}{3}, 1$ and $\frac{2}{3}$, leading to the percentages of $16.7 \%, 50 \%$ and $33.3 \%$; the expected numbers of $N, T$, and $F$ in period 2 are respectively $\frac{2}{3}, 1$ and $\frac{1}{3}$, leading to the percentages of $33.3 \%, 50 \%$ and $16.7 \%$; the expected total industry profit is $\frac{1}{3} \times\left(\pi_{1}^{\{\{N, T\},\{F, T\}\}}+\pi_{2}^{\{\{N, T\},\{F, T\}\}}\right)+\frac{2}{3} \times\left(\pi_{1}^{\{\{T, F\},\{N, T\}\}}+\pi_{2}^{\{\{T, F\},\{N, T\}\}}\right)$, where $\pi_{i}^{\left\{\left\{a_{1}, a_{2}\right\},\left\{b_{1}, b_{2}\right\}\right\}}$ denotes store $i$ 's profit under equilibrium $\left\{\left\{a_{1}, a_{2}\right\},\left\{b_{1}, b_{2}\right\}\right\}$.

Figure 2 shows how occurrences of $N, T$, and $F$ change with $d$. These plots are obtained by averaging across all equilibrium cases with a particular value of $d$. We observe jumps at $d=0.5$ because equilibrium behavior differs across the two regions divided by $d .{ }^{8}$ More generally, as $d$ increases, in both periods, stores are more likely to adopt $N$ and $F$, and less likely to adopt $T$. Hence, false promotions are more prevalent in both periods when the market is less competitive, although we see false promotions are more likely to take place in the first period, whereas real promotions and no promotions are more likely to take place in the second period. We find this somewhat counter-intuitive in that there is no long-term penalty for offering false promotions in the second period. We conjecture that this is due, in large part, to the fact that by offering false promotions early on, the firms have "poisoned" the well and thus the larger sophisticated population resulting from the subsequent WOM dampens the viability of offering false promotions in later periods.

\footnotetext{
${ }^{8}$ When $d<0.5$, if one store chooses $F$, then $N$ is dominated by $T$ for the other store, so only $T$ or $F$ can be the optimal strategy. In contrast, when $d>0.5$, this is no longer the case and $N$ can also be the optimal strategy when the other store choooses $F$, leading to different equilibrium outcomes.
} 

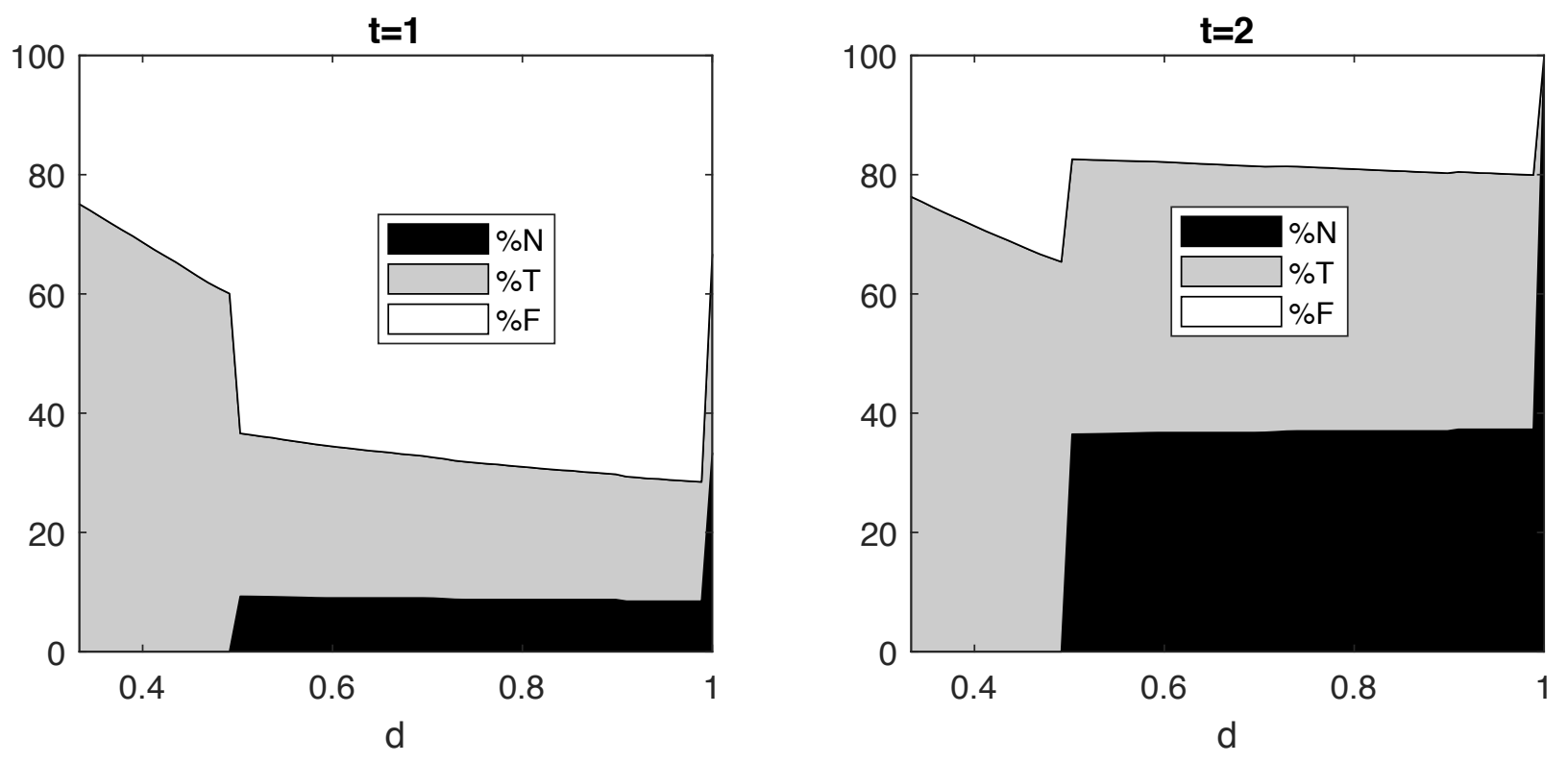

Figure 2: Effects of $d$ on Promotional Activities

In Figure 3 we show the effects of $\lambda+\epsilon$ (maximum fraction of sophisticated consumers) on the occurrences of $N, T$, and $F$. As $\lambda+\epsilon$ increases, false promotions become less prevalent and real promotions become more prevalent in both periods. We find it interesting that high values of $\lambda+\epsilon$ do not insure that there are no false promotions. We believe this is due to the fact that by giving a false promotion, the firm is able to get out of the prisoner's dilemma.
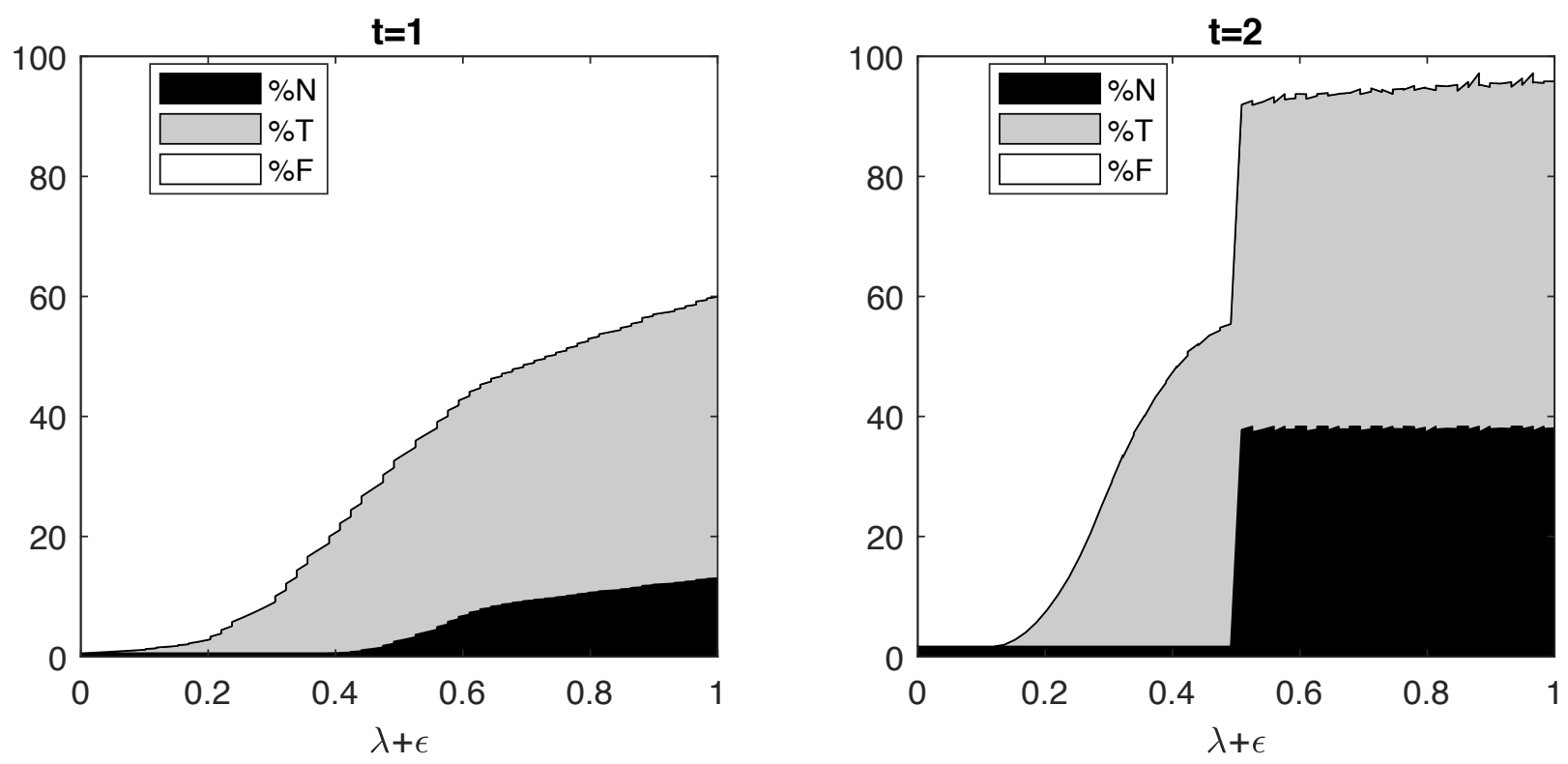

Figure 3: Effects of $\lambda+\epsilon$ on Promotional Activities 
We next investigate the impact of the option to offer false promotions on industry profits. Figure 4 shows how $d$ affects the baseline total profit when false promotions are not allowed, total profit with the option of false promotions, the difference between total profit and baseline profit, and the difference normalized by baseline profit. As $d$ increases, profits with and without the option of false promotions both increase, and their gap shrinks. Hence, the net increase in total profits is greatest in highly competitive settings and that there is again a major shift after $d>0.5$, indicating the advantage of having an option to offer false promotions decreases as competition softens.

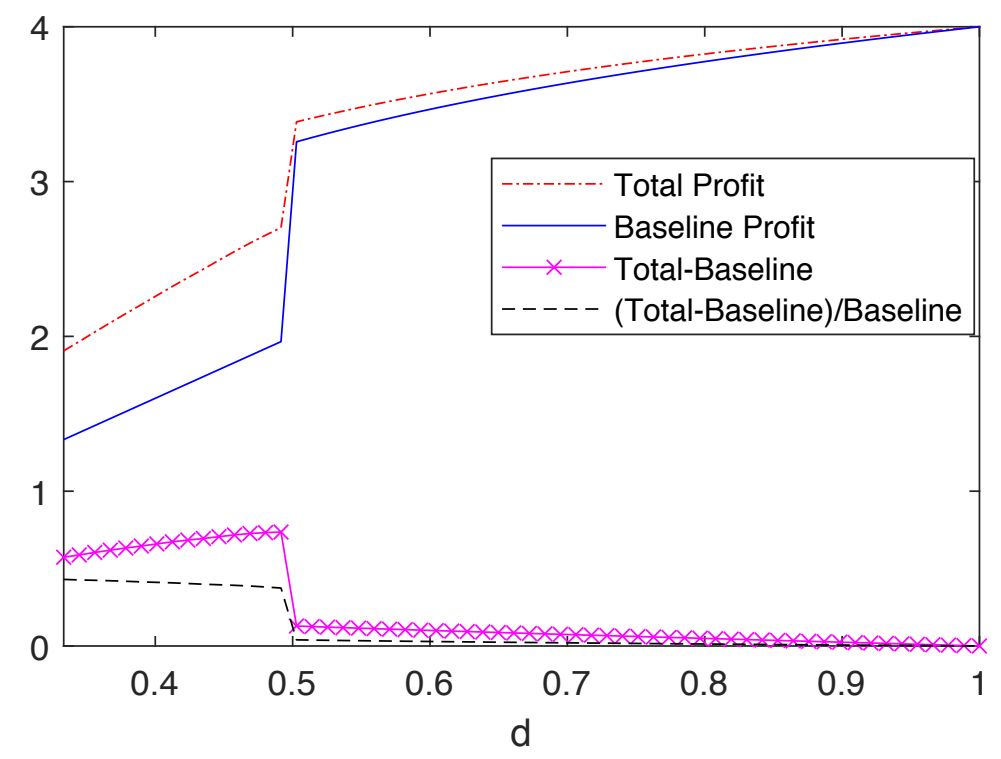

Figure 4: Effect of $d$ on Total Industry Profits

The effects of $\lambda+\epsilon$ on industry profits (Figure 5) are as expected. Thus, increases in the maximum fraction of sophisticated consumers decrease the opportunity to increase total industry profits by adding false promotions into the strategy space. 


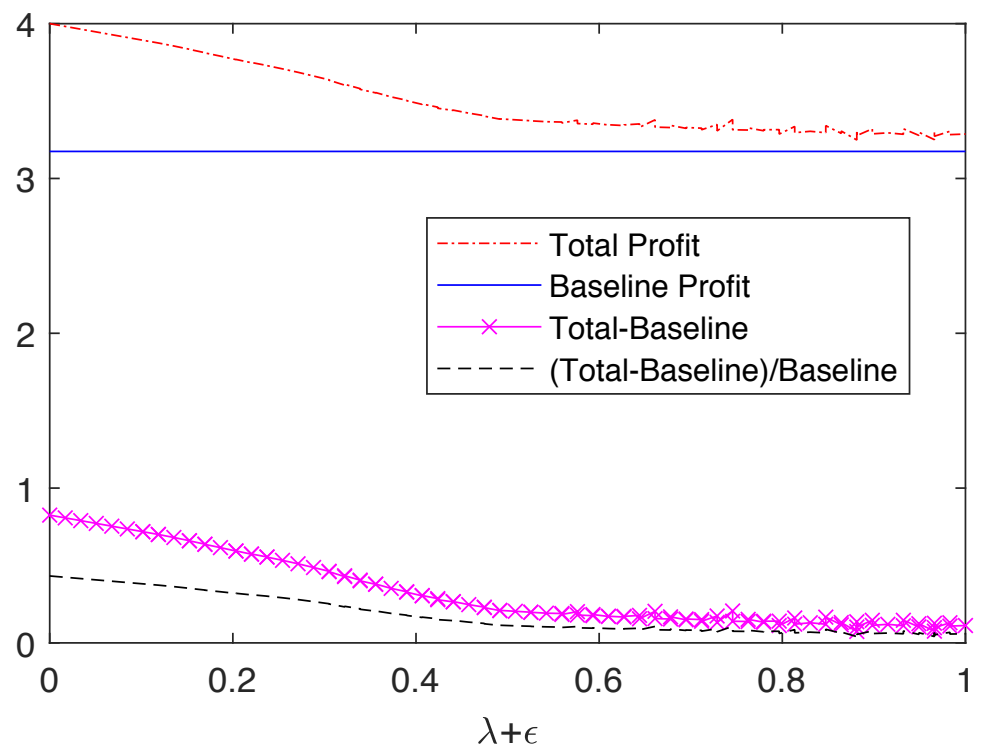

Figure 5: Effect of $\lambda+\epsilon$ on Total Industry Profits

\section{Discussions}

\subsection{Alternative Utility Specifications}

Our specification of consumer utility is based on Thaler's transaction utility theory. We next explore implications if consumer utility takes alternative forms. We do this by comparing our specification with three alternative specifications: the classical linear utility ("Classical"), the referencedependent preferences (e.g., Heidhues and Köszegi, 2008) ("Within-store reference-dependence"), and the reference-dependent preferences where prices of both firms are taken into account (e.g., Ray et al., 2015) ("Industry reference-dependence").

\subsubsection{Classical}

The classical linear utility is specified as follows:

$$
u_{i}=v-p_{i}-x_{i},
$$

where $p_{i}$ is the actual retail price of store $i$, and $x_{i}$ is the travel distance to store $i . p_{i}=p^{\star}$ if store $i$ chooses $N$ or $F$, and $p_{i}=\left(1-\alpha_{i}\right) p^{\star}$ if store $i$ chooses $T$, where $\alpha_{i}$ is the optimal discount rate of store $i$ and is endogenously determined. 
Column (1) of Table 5 summarizes utilities in all promotion strategy profiles under the classical approach. ${ }^{9}$

Table 5: Utility Comparison

\begin{tabular}{|c|c|c|c|c|c|}
\hline $\begin{array}{l}\text { Promoti } \\
\text { Profile }\end{array}$ & AStrategy & $\begin{array}{l}\text { (1) Classical (Eq } \\
(2))\end{array}$ & (2) Our approach & $\begin{array}{l}(3) \text { Within-store } \\
\text { reference- } \\
\text { dependence (Eq } \\
(3))\end{array}$ & $\begin{array}{l}(4) \text { Industry } \\
\text { reference- } \\
\text { dependence (Eq } \\
(5))\end{array}$ \\
\hline \multirow{2}{*}{$N, N$} & $u_{1}$ & $v-p^{\star}-x_{1}$ & $v-p^{\star}-x_{1}$ & $v-p^{\star}-x_{1}$ & $v-p^{\star}-x_{1}$ \\
\hline & $u_{2}$ & $v-p^{\star}-x_{2}$ & $v-p^{\star}-x_{2}$ & $v-p^{\star}-x_{2}$ & $v-p^{\star}-x_{2}$ \\
\hline \multirow{3}{*}{$N, T$} & $u_{1}$ & $v-p^{\star}-x_{1}$ & $v-p^{\star}-x_{1}$ & $v-p^{\star}-x_{1}$ & $v-p^{\star}-x_{1}$ \\
\hline & $u_{2}$ & $v-\left(1-\alpha_{2}\right) p^{\star}-$ & $v-\left(1-\alpha_{2}\right) p^{\star}-$ & $v-\left(1-\alpha_{2}\right) p^{\star}-$ & $v-\left(1-\alpha_{2}\right) p^{\star}-$ \\
\hline & & $x_{2}$ & $x_{2}+\alpha_{2} v$ & $x_{2}+\alpha_{2} v$ & $x_{2}+\alpha_{2} v$ \\
\hline \multirow{3}{*}{$N, F$} & $u_{1}$ & $v-p^{\star}-x_{1}$ & $v-p^{\star}-x_{1}$ & $v-p^{\star}-x_{1}$ & $v-p^{\star}-x_{1}$ \\
\hline & $u_{s 2}$ & $v-p^{\star}-x_{2}$ & $v-p^{\star}-x_{2}-\alpha_{2} v$ & $v-p^{\star}-x_{2}$ & $v-p^{\star}-x_{2}$ \\
\hline & $u_{n 2}$ & $v-p^{\star}-x_{2}$ & $v-p^{\star}-x_{2}+\alpha_{2} v$ & $v-p^{\star}-x_{2}+\alpha_{2} v$ & $v-p^{\star}-x_{2}$ \\
\hline \multirow{4}{*}{$T, T$} & $u_{1}$ & $v-\left(1-\alpha_{1}\right) p^{\star}-$ & $v-\left(1-\alpha_{1}\right) p^{\star}-$ & $v-\left(1-\alpha_{1}\right) p^{\star}-$ & $v-\left(1-\alpha_{1}\right) p^{\star}-$ \\
\hline & & $x_{1}$ & $x_{1}+\alpha_{1} v$ & $x_{1}+\alpha_{1} v$ & $x_{1}$ \\
\hline & $u_{2}$ & $v-\left(1-\alpha_{2}\right) p^{\star}-$ & $v-\left(1-\alpha_{2}\right) p^{\star}-$ & $v-\left(1-\alpha_{2}\right) p^{\star}-$ & $v-\left(1-\alpha_{2}\right) p^{\star}-$ \\
\hline & & $x_{2}$ & $x_{2}+\alpha_{2} v$ & $x_{2}+\alpha_{2} v$ & $x_{2}$ \\
\hline \multirow{4}{*}{$T, F$} & $u_{1}$ & $v-\left(1-\alpha_{1}\right) p^{\star}-$ & $v-\left(1-\alpha_{1}\right) p^{\star}-$ & $v-\left(1-\alpha_{1}\right) p^{\star}-$ & $v-\left(1-\alpha_{1}\right) p^{\star}-$ \\
\hline & & $x_{1}$ & $x_{1}+\alpha_{1} v$ & $x_{1}+\alpha_{1} v$ & $x_{1}+\alpha_{1} v$ \\
\hline & $u_{s 2}$ & $v-p^{\star}-x_{2}$ & $v-p^{\star}-x_{2}-\alpha_{2} v$ & $v-p^{\star}-x_{2}$ & $v-p^{\star}-x_{2}$ \\
\hline & $u_{n 2}$ & $v-p^{\star}-x_{2}$ & $v-p^{\star}-x_{2}+\alpha_{2} v$ & $v-p^{\star}-x_{2}+\alpha_{2} v$ & $v-p^{\star}-x_{2}$ \\
\hline \multirow{4}{*}{$F, F$} & $u_{s 1}$ & $v-p^{\star}-x_{1}$ & $v-p^{\star}-x_{1}-\alpha_{1} v$ & $v-p^{\star}-x_{1}$ & $v-p^{\star}-x_{1}$ \\
\hline & $u_{n 1}$ & $v-p^{\star}-x_{1}$ & $v-p^{\star}-x_{1}+\alpha_{1} v$ & $v-p^{\star}-x_{1}+\alpha_{1} v$ & $v-p^{\star}-x_{1}$ \\
\hline & $u_{s 2}$ & $v-p^{\star}-x_{2}$ & $v-p^{\star}-x_{2}-\alpha_{2} v$ & $v-p^{\star}-x_{2}$ & $v-p^{\star}-x_{2}$ \\
\hline & $u_{n 2}$ & $v-p^{\star}-x_{2}$ & $v-p^{\star}-x_{2}+\alpha_{2} v$ & $v-p^{\star}-x_{2}+\alpha_{2} v$ & $v-p^{\star}-x_{2}$ \\
\hline
\end{tabular}

Let $D:=(v+p) d$. The table below summarizes the stage game under this specification:

Store 2

\begin{tabular}{|c|c|c|c|c|}
\hline & & $N$ & $T$ & $F$ \\
\hline \multirow{3}{*}{ Store 1} & $N$ & 1,1 & $\frac{3 D-p^{\star}}{2 D d}, \frac{\left(D+p^{\star}\right)^{2}}{4 p^{\star} D}$ & 1,1 \\
\hline & $T$ & $\frac{\left(D+p^{\star}\right)^{2}}{4 p^{\star} D}, \frac{3 D-p^{\star}}{2 D d}$ & $\frac{D}{p^{\star}}, \frac{D}{p^{\star}}$ & $\frac{\left(D+p^{\star}\right)^{2}}{4 p^{\star} D}, \frac{3 D-p^{\star}}{2 D d}$ \\
\hline & $F$ & 1,1 & $\frac{3 D-p^{\star}}{2 D d}, \frac{\left(D+p^{\star}\right)^{2}}{4 p^{\star} D}$ & 1,1 \\
\hline
\end{tabular}

The classical utility function implies no extra gain from adopting false promotions as compared with no promotion. This leads to $T$ being a dominant strategy for both stores. Hence, the prisoner's

\footnotetext{
${ }^{9}$ To conserve space, we omit three strategy profiles $(T, N),(F, T)$ and $(F, N)$ which are symmetric to $(N, T)$, $(T, F)$ and $(N, F)$ respectively. When store $i$ chooses $T$ or $N$, the utility of sophisticated consumers and that of naive consumers are the same, which we denote with $u_{i}$. When store $i$ chooses $F$, sophisticated consumers obtain utility $u_{s i}$ whereas naive consumers obtain utility $u_{n i}$, and the two utilities may differ depending on the utility specification.
} 
dilemma cannot be avoided. Because $\lambda$ is not involved in the stage game, WOM is irrelevant. Further, because there is no belief updating, the game reduces to a two-period repeated game where the only SPNE is $\{\{T, T\},\{T, T\}\}$. Therefore, the classical specification is not suitable for addressing our issue.

\subsubsection{Within-store Reference-dependence}

The standard reference-dependent utility is specified as follows:

$$
u_{i}=v-p_{i}-x_{i}+I\left(p_{r i}, p_{i}\right) \times\left(\frac{p_{r i}-p_{i}}{p_{r i}}\right) v
$$

where $p_{i}$ is defined similarly as in Equation (2). $p_{r i}$ is the reference price associated with store $i$, which we take as the perceived list price. When store $i$ chooses $T, p_{r i}=p^{\star}$ and $p_{i}=\left(1-\alpha_{i}\right) p^{\star}$. When store $i$ chooses $N, p_{r i}=p_{i}=p^{\star}$. When store $i$ chooses $F, p_{i}=p^{\star}$, and $p_{r i}=p^{\star}$ for sophisticated consumers and $p_{r i}=\frac{p^{\star}}{1-\alpha_{i}}$ for naive consumers. $I\left(p_{r i}, p_{i}\right)$ is an indicator function that equals 1 if $p_{r i}>p_{i}$ and 0 otherwise. We denote this specification as "within-store referencedependence" because the reference price is independent of the other store's price.

Therefore, under this utility specification, when store $i$ chooses $T$, consumers obtain utility $u_{i}=v-\left(1-\alpha_{i}\right) p^{\star}-x_{i}+\left(\frac{p^{\star}-\left(1-\alpha_{i}\right) p^{\star}}{p^{\star}}\right) v=v-\left(1-\alpha_{i}\right) p^{\star}-x_{i}+\alpha_{i} v$. When store $i$ chooses $N$, consumers obtain utility $u_{i}=v-p^{\star}-x_{i}$. When store $i$ chooses $F$, sophisticated consumers obtain

utility $u_{s i}=v-p^{\star}-x_{i}$ whereas naive consumers obtain utility $u_{n i}=v-p^{\star}-x_{i}+\left(\frac{\frac{p^{\star}}{1-\alpha_{i}}-p^{\star}}{\frac{p^{\star}}{1-\alpha_{i}}}\right) v=$ $v-p^{\star}-x_{i}+\alpha_{i} v$. Column (3) of Table 5 summarizes utilities in all promotion strategy profiles under the standard reference-dependent specification.

The above implies that under this standard reference-dependent specification, when a false promotion is adopted, there is no utility loss, even for sophisticated consumers. Hence, false promotions are more beneficial in this case than in our model. All our results hold qualitatively in this case.

Extension to accommodate false promotions It is possible to modify the standard referencedependent specification to accommodate false promotions and make it equivalent to our utility 
specification. We do so by adding a term $-\left(\frac{p_{c i}-p_{r i}}{p_{c i}}\right) v$ to Equation (3):

$$
u_{i}=v-p_{i}-x_{i}+I\left(p_{r i}, p_{i}\right) \times\left(\frac{p_{r i}-p_{i}}{p_{r i}}\right) v-\left(\frac{p_{c i}-p_{r i}}{p_{c i}}\right) v
$$

where $p_{c i}$ is the list price claimed by store $i$. Note that the new term is different from zero (i.e., $\left.p_{c i} \neq p_{r i}\right)$ only for sophisticated consumers when store $i$ offers a false promotion. In this case, the claimed list price of store $i$ is $p_{c i}=\frac{p^{\star}}{1-\alpha_{i}}$. However, a sophisticated consumer knows the true list price is $p^{\star}$ and takes this price as the reference price: $p_{r i}=p^{\star}$. It is easy to show that this utility function is equivalent to the utility function in our model, where utilities under all promotion strategy profiles are shown in Column (2) of Table 5. For instance, when store $i$ chooses $F$, for sophisticated consumers, $I\left(p_{r i}, p_{i}\right)=0, p_{c i} \neq p_{r i}$, and utility is $u_{s i}=v-p^{\star}-x_{i}-$ $\left(\frac{\frac{p^{\star}}{1-\alpha_{i}}-p^{\star}}{\frac{p^{\star}}{1-\alpha_{i}}}\right) v=v-p^{\star}-x_{i}-\alpha_{i} v$. However, for naive consumers, $I\left(p_{r i}, p_{i}\right)=1, p_{c i}=p_{r i}$, and utility is $u_{n i}=v-p^{\star}-x_{i}+\left(\frac{\frac{p^{\star}}{1-\alpha_{i}}-p^{\star}}{\frac{p^{\star}}{1-\alpha_{i}}}\right) v=v-p^{\star}-x_{i}+\alpha_{i} v$.

\subsubsection{Industry Reference-dependence}

Following Ray et al. (2015), we specify as follows the reference-dependent utility where prices of both firms are taken into account:

$$
u_{i}=v-p_{i}-x_{i}+I\left(p_{i}, p_{j}\right) \times\left(\frac{p_{j}-p_{i}}{p_{j}}\right) v,
$$

where $p_{i}$ is defined similarly as in Equation (2), and $p_{j}$ is the actual price of the other store. $I\left(p_{i}, p_{j}\right)=1$ if $p_{i}<p_{j}$ and $I\left(p_{i}, p_{j}\right)=0$ otherwise. Note that this utility specification differs from the classical utility function only when the two stores offer different prices, i.e., one and only one store chooses $T$. For example, when store 1 chooses $N$ or $F$ and store 2 chooses $T$, consumers obtain utility $u_{1}=v-p^{\star}-x_{1}$ from store 1 , and utility $u_{2}=v-\left(1-\alpha_{2}\right) p^{\star}-x_{2}+\left(\frac{p^{\star}-\left(1-\alpha_{2}\right) p^{\star}}{p^{\star}}\right) v=$ $v-\left(1-\alpha_{2}\right) p^{\star}-x_{2}+\alpha_{2} v$ from store 2 . We denote this specification as "industry referencedependence" because the other store's price is taken as the reference price. Column (4) of Table 5 summarizes utilities in all promotion strategy profiles under this specification, and the table below summarizes the stage game: 
Store 2

\begin{tabular}{cc|c|c|c|} 
& \multicolumn{2}{c}{$N$} & \multicolumn{2}{c}{$T$} \\
\cline { 3 - 5 } & $N$ & 1,1 & $\frac{3 d-1}{2 d}, \frac{(1+d)^{2}}{4 d}$ & 1,1 \\
\cline { 3 - 5 } Store 1 & $T$ & $\frac{(1+d)^{2}}{4 d}, \frac{3 d-1}{2 d}$ & $\frac{D}{p^{\star}}, \frac{D}{p^{\star}}$ & $\frac{(1+d)^{2}}{4 d}, \frac{3 d-1}{2 d}$ \\
\cline { 3 - 5 } & & 1,1 & $\frac{3 d-1}{2 d}, \frac{(1+d)^{2}}{4 d}$ & 1,1 \\
\cline { 3 - 5 } & $F$ & 1,1 & &
\end{tabular}

This industry reference-dependent specification is close to the classical specification because the extra profit gain is very limited. In particular, false promotions induce extra profit gains only when the other store chooses $T$. This extra profit in this case is not large enough for $F$ to dominate $T$ under some circumstances. Therefore, as under the classical specification, $T$ is a dominant strategy for both stores, and the only SPNE is $\{\{T, T\},\{T, T\}\}$.

Extension to accommodate false promotions Similar to the standard reference-dependent specification, under the industry reference-dependent specification described above, when a false promotion is adopted, there is no utility loss. We can also modify this specification to accommodate false promotions by adding a term $-\left(\frac{p_{c i}-p_{r i}}{p_{c i}}\right) v$ to Equation (5). In addition, we replace $p_{j}$ with $p_{r i}^{\prime}$, the new reference price based on both stores' prices. The utility function is therefore as follows:

$$
u_{i}=v-p_{i}-x_{i}+I\left(p_{i}, p_{r i}^{\prime}\right) \times\left(\frac{p_{r i}^{\prime}-p_{i}}{p_{r i}^{\prime}}\right) v-\left(\frac{p_{c i}-p_{r i}^{\prime}}{p_{c i}}\right) v
$$

where $p_{c i}$ is the list price claimed by store $i$, and $p_{r i}^{\prime}$ is the reference price associated with store $i$. However, $p_{r i}^{\prime}$ is different from $p_{r i}$ in Equation (3) because it should also take into account the other store's actual and claimed prices. One way to do this is to set the reference price as a weighted average of $p_{r 1}$ and $p_{r 2}$ in Equation (3). Without loss of generality, assume $p_{r i}^{\prime}=\frac{p_{r 1}+p_{r 2}}{2}, i=1,2$. Under this specification:

1. When neither store chooses $F, p_{r i}^{\prime}=p^{\star}$, and the utilities are the same as under our model specification.

2. When both stores choose $F, p_{r i}^{\prime}=p^{\star}$ for sophisticated consumers, and $p_{r i}^{\prime}=\frac{p^{\star}}{1-\alpha_{i}}$ for naive consumers. Hence, the utilities are also the same as under our model specification.

3. When store 1 chooses $N$ or $T$ and store 2 chooses $F$, for sophisticated consumers, $p_{r 1}^{\prime}=p_{r 2}^{\prime}=$ $p^{\star}, p_{c 1}=p_{r 1}^{\prime}, p_{c 2} \neq p_{r 2}^{\prime}$, so that their utility is the same as under our model specification. In 
contrast, naive consumers' utility is different from that under our model specification. More specifically, $p_{r 1}^{\prime}=p_{r 2}^{\prime}=\frac{p^{\star}+\frac{p^{\star}}{1-\alpha_{2}}}{2}=\left(1+\frac{\alpha_{2}}{2\left(1-\alpha_{2}\right)}\right) p^{\star}, p_{c 1} \neq p_{r 1}^{\prime}, p_{c 2} \neq p_{r 2}^{\prime}$, so that their utility from store 2 is $u_{n 2}=v-p^{\star}-x_{2}+\left(\frac{\left(1+\frac{\alpha_{2}}{2\left(1-\alpha_{2}\right)}\right) p^{\star}-p^{\star}}{\left(1+\frac{\alpha_{2}}{2\left(1-\alpha_{2}\right)}\right) p^{\star}}\right) v-\left(\frac{\frac{p^{\star}}{1-\alpha_{2}}-\left(1+\frac{\alpha_{2}}{2\left(1-\alpha_{2}\right)}\right) p^{\star}}{\frac{p^{\star}}{1-\alpha_{2}}}\right) v=$ $v-p^{\star}-x_{2}+\frac{\alpha_{2}}{2-\alpha_{2}} v-\frac{\alpha_{2}}{2} v$. When store 1 chooses $N, u_{n 1}=v-p^{\star}-x_{1}+\left(\frac{\left(1+\frac{\alpha_{2}}{2\left(1-\alpha_{2}\right)}\right) p^{\star}-p^{\star}}{\left(1+\frac{\alpha_{2}}{2\left(1-\alpha_{2}\right)}\right) p^{\star}}\right) v-$ $\left(\frac{p^{\star}-\left(1+\frac{\alpha_{2}}{2\left(1-\alpha_{2}\right)}\right) p^{\star}}{p^{\star}}\right) v=v-p^{\star}-x_{1}+\frac{\alpha_{2}}{2-\alpha_{2}} v+\frac{\alpha_{2}}{2\left(1-\alpha_{2}\right)} v$, and when store 1 chooses $T, u_{n 1}=$ $v-\left(1-\alpha_{1}\right) p^{\star}-x_{1}+\left(\frac{\left(1+\frac{\alpha_{2}}{2\left(1-\alpha_{2}\right)}\right) p^{\star}-\left(1-\alpha_{1}\right) p^{\star}}{\left(1+\frac{\alpha_{2}}{2\left(1-\alpha_{2}\right)}\right) p^{\star}}\right) v-\left(\frac{p^{\star}-\left(1+\frac{\alpha_{2}}{2\left(1-\alpha_{2}\right)}\right) p^{\star}}{p^{\star}}\right) v=v-p^{\star}-x_{1}+$ $\frac{2 \alpha_{1}+\alpha_{2}-2 \alpha_{1} \alpha_{2}}{2-\alpha_{2}} v+\frac{\alpha_{2}}{2\left(1-\alpha_{2}\right)} v$. In words, under this formulation, when naïve consumers see a promotion, they give positive utility to the firm offering the promotion. However, they also make inferences about the firm based on its list price relative to the reference price. Firms with high list prices are punished if their list price is greater than the industry average.

In sum, the specification in Equation (6) leads to same utilities as under our original specification in most cases. Utilities only differ for naive consumers when one and only one store chooses $F$, in which case the benefit associated with a false promotion decreases and the benefit associated with a real promotion increases. Our main results hold qualitatively under this specification.

\section{$5.2 \quad$ No WOM}

If there is no WOM, then the population of sophisticated consumers is constant over time. Consequently, the dynamic game in our model reduces to a two-period repeated game, and more than half of SPNEs in the model would be eliminated.

Table 6 summarizes all SPNEs when there is no WOM when $d<0.5 .^{10}$ The case numbers correspond to those in Table 2.

Table 6: SPNEs without WOM when $d<0.5$

\begin{tabular}{clc}
\hline Case & Condition & SPNE \\
\hline 1 & $\lambda+\epsilon<\min \{\underline{\theta}, \bar{\theta}\}$ & $\{\{F, F\},\{F, F\}\}$ \\
4 & $\underline{\theta}<\lambda<\lambda+\epsilon<\bar{\theta}$ & $\{\{F, T\},\{F, T\}\},\{\{T, F\},\{T, F\}\}$, \\
& & $\{\{T, F\},\{F, T\}\},\{\{F, T\},\{T, F\}\}$ \\
5 & $\bar{\theta}<\lambda<\lambda+\epsilon<\underline{\theta}$ & $\{\{T, T\},\{T, T\}\},\{\{F, F\},\{T, T\}\}$, \\
& & $\{\{T, T\},\{F, F\}\},\{\{F, F\},\{F, F\}\}$ \\
9 & $\lambda>\max \{\underline{\theta}, \bar{\theta}\}$, & $\{\{T, T\},\{T, T\}\}$ \\
\hline
\end{tabular}

\footnotetext{
${ }^{10}$ Proofs are available upon request.
} 
As we can see, Cases 2, 3, 6, 7, and 8 in Table 2 are eliminated. Intuitively, when there is no WOM, the only factor that governs the punishment of adopting false promotions in the first period is the population of sophisticated consumers. Hence, SPNEs that require more punishment over the two periods than the population of sophisticated consumers provides in the first period can no longer be SPNEs. For instance, in Case 2 of Table 2, there are few sophisticated consumers and medium WOM. Both stores tend to use false promotion in the first period as most consumers can be fooled. However, sophisticated consumers expand due to WOM in the second period. The WOM effect is medium such that false promotions, if used by one store, is still profitable. If there is no WOM, however, then adopting false promotions in both periods is optimal for both stores. Hence, these SPNEs do not exist without WOM.

Table 7 summarizes all SPNEs when there is no WOM when $d \geq 0.5$, replicating the numerical analysis described in $\S 4.4$. The case numbers correspond to those in Table 4, and we can see that Cases 2, 3, 5, and 6 are eliminated.

Table 7: SPNEs when $d \geq 0.5$

\begin{tabular}{cll}
\hline Case & SPNE & Occurrence \\
\hline 1 & $\{\{F, F\},\{F, F\}\}$ & $32.6 \%$ \\
4 & $\{\{T, F\},\{T, F\}\},\{\{T, F\},\{F, T\}\}$, & $17.4 \%$ \\
& $\{\{F, T\},\{T, F\}\},\{\{F, T\},\{F, T\}\}$ & \\
7 & $\{\{N, T\},\{N, T\}\},\{\{N, T\},\{T, N\}\}$, & $49.0 \%$ \\
& $\{\{T, N\},\{N, T\}\},\{\{T, N\},\{T, N\}\}$, & \\
& $\{\{T, F\},\{N, T\}\},\{\{F, T\},\{T, N\}\}$, & $1.0 \%$ \\
$8 \quad$ & $\{\{N, T\},\{N, T\}\},\{\{N, T\},\{T, N\}\}$, & \\
& $\{\{T, N\},\{N, T\}\},\{\{T, N\},\{T, N\}\}$, & \\
& $\{\{T, T\},\{N, T\}\},\{\{T, T\},\{T, N\}\}$, & \\
& $\{\{T, F\},\{N, T\}\},\{\{F, T\},\{T, N\}\}$ & \\
\hline
\end{tabular}

Figure 6 replicates Figure 2 and shows how occurrences of $N, T$, and $F$ change with $d$ when there is no WOM. The general patterns are similar to those in Figure 2. However, when there is no WOM, we observe fewer false promotions in the first period and more false promotions in the second period. 

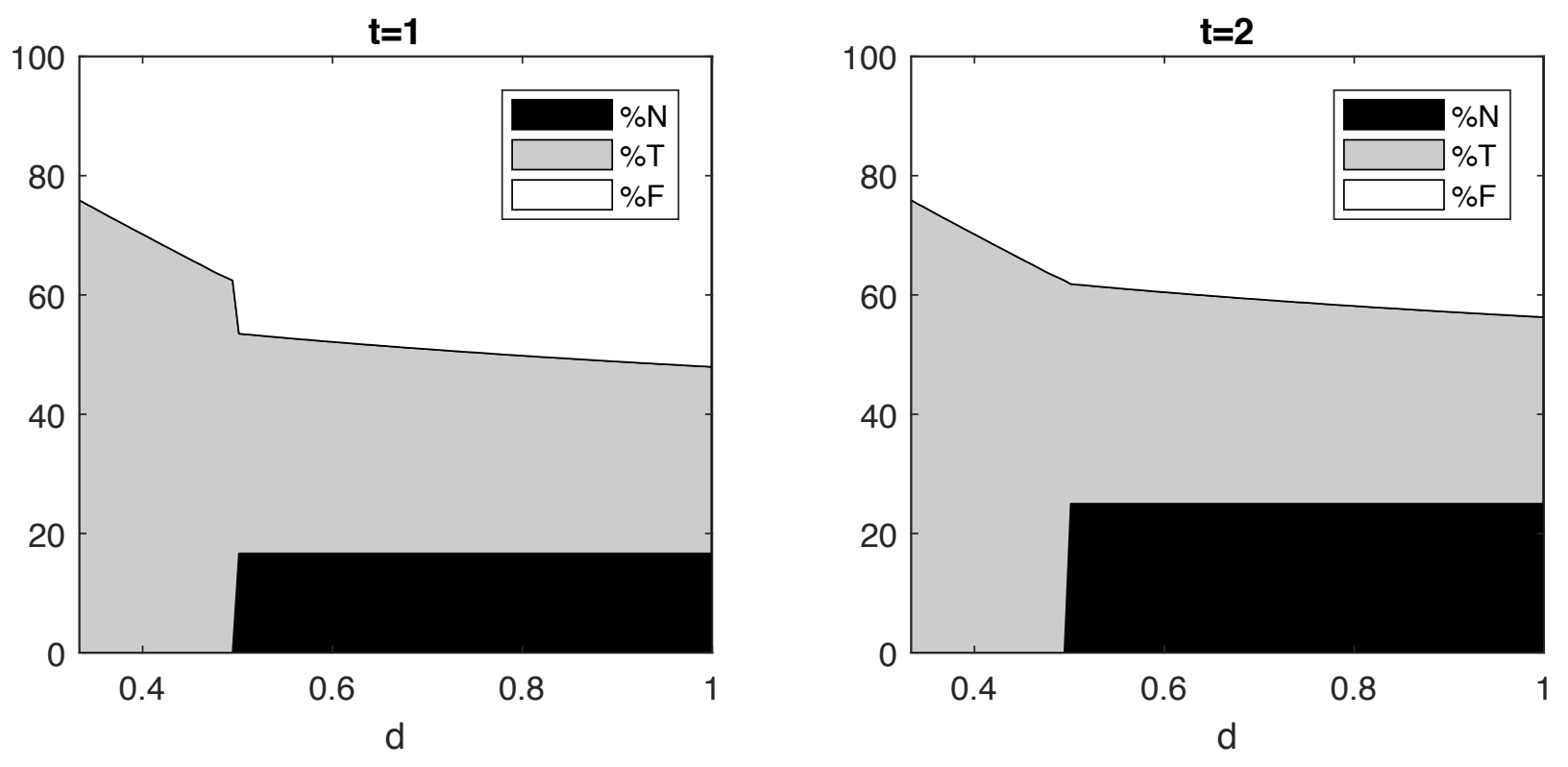

Figure 6: Effects of $d$ on Promotional Activities Without WOM

\subsection{Equilibrium Selection Based on Turn-Taking Equilibrium}

So far we have not considered equilibrium selection. There is a literature on two players taking turns to determine strategies for both parties over time (e.g., Durant et al., Forthcoming), and we use this approach for equilibrium selection. Without loss of generality, assume store $i$ is the decision maker in the $i$ th period. In this environment, store $i$ selects the Nash equilibrium that maximizes its payoff in the $i$ th period. This SPNE is defined as a turn-taking equilibrium (TTE). Therefore, in each case in Table 2, there is only one TTE. Table 8 lists all the TTEs. ${ }^{11}$

Table 8: SPNEs Under TTE

\begin{tabular}{clc}
\hline Case & Condition & SPNE \\
\hline 1 & $\lambda+\epsilon<\min \{\underline{\theta}, \bar{\theta}\}$ & $\{\{F, F\},\{F, F\}\}$ \\
2 & $\lambda<\underline{\theta}<\lambda+\epsilon<\bar{\theta}$ & $\{\{F, F\},\{F, T\}\}$ \\
3 & $\lambda<\bar{\theta}<\lambda+\epsilon<\underline{\theta}$ & $\{\{F, F\},\{F, F\}\}$ \\
4 & $\underline{\theta}<\lambda<\lambda+\epsilon<\bar{\theta}$ & $\{\{T, F\},\{F, T\}\}$ \\
5 & $\bar{\theta}<\lambda<\lambda+\epsilon<\underline{\theta}$ & $\{\{F, F\},\{F, F\}\}$ \\
6 & $\lambda<\min \{\underline{\theta}, \bar{\theta}\}<\max \{\underline{\theta}, \bar{\theta}\}<\lambda+\epsilon$ & $\{\{F, F\},\{T, T\}\}$ \\
7 & $\underline{\theta}<\lambda<\bar{\theta}<\lambda+\epsilon$ & $\{\{T, F\},\{T, T\}\}$ \\
8 & $\bar{\theta}<\lambda<\underline{\theta}<\lambda+\epsilon$ & $\{\{F, F\},\{T, T\}\}$ \\
9 & $\lambda>\max \{\underline{\theta}, \bar{\theta}\}$ & $\{\{T, T\},\{T, T\}\}$ \\
\hline
\end{tabular}

\footnotetext{
${ }^{11}$ Proofs are available upon request.
} 
Intuitively, when the share of sophisticated consumers is small and the WOM effect is small, adopting false promotions in both periods is the most profitable strategy for both stores. As these two factors become larger, stores have to adopt more real promotions in equilibrium. Notice that both stores always prefer equilibria that involve false promotions as long as the market share of

sophisticated consumer is not large enough $(\lambda<\max \{\underline{\theta}, \bar{\theta}\}$ and $\lambda+\epsilon<\max \{\underline{\theta}, \bar{\theta}\}$ for store 1 and store 2, respectively). This result is consistent with Durant et al. (Forthcoming) in that, compared to our baseline framework, turn-taking increases false promotions (collusion/cooperation) in equilibrium.

\section{Conclusions}

This paper addresses the question "under what conditions would a firm give a 'false' promotion and with what regularity". We do this by developing and solving a model that incorporates forwardlooking retailers, retailer competition and WOM. These retailers sell into a market composed of two types of consumers, sophisticated consumers who can detect false promotions and naive consumers who cannot. The sophisticated consumers pass on information about the existence of false promotions to a subset of naive consumers, thereby increasing the pool of sophisticated consumes in the next period.

We find when the market is competitive, the possibility of offering false promotions can improve the payoffs for both firms. This is counter-intuitive, and the rationale is as follows. When false promotions are not available as an option, a real promotion is always the best response when the other firm provides a real promotion, leading to the prisoner's dilemma. When false promotions become an option, a real promotion is no longer the best response to a real promotion under certain circumstances. Even at lower levels of competition where the prisoner's dilemma is no longer an equilibrium solution without false promotions, we still find on average, total industry profits increase over all levels of competition. We also find that even when consumers can identify that a firm is offering a false promotion, and thus not only stay away from the falsely promoted offering from the firm, but also pass this information to others, a firm still finds it optimal to offer such promotions from time to time. Surprisingly false promotions are more likely to occur in the first period compared to the second period. Thus, the longer-term impact of increasing the magnitude of knowledgeable consumers does not seem to deter the firms from offering false promotions. However, 
as expected, we find in general, that a firm is less likely to offer false promotions in an environment with more sophisticated consumers and stronger WOM. In fact, if the market is initially composed of at least $50 \%$ sophisticated consumers, we observe no false promotions in the final period. In addition, when competition is high and the pool of sophisticated consumers is large, we still find firms caught in the prisoner's dilemma even when giving false promotions is an option. Finally, our model offers explanations to both the within-store and between-store false promotions.

Our analyses are based on the practice of giving false price promotions. However, the model is general enough to explain the existence of a number of deceptive marketing practices detailed by Lindsey-Mullikin and Petty (2011). For instance, when a retailer makes a false (dubious) claim regarding product quality, naive consumers tend to get lured while sophisticated consumers get upset. Retailers can also list false reference prices (both their own and their rivals'). In these situations it is often difficult for regulators to act upon various false claims because retailers are able to take advantage of legal loopholes.

It is interesting to note that it may not be in the industry's best interest to "clean itself up", in that the existence of deceptive marketing practices may lead to higher industry profits, especially when the market is more competitive. This will occur even in situations where the knowledgeable consumers can pass on their knowledge to a subset of the consumers previously deceived by these dubious practices. This raises the issue of public policy since it does not necessarily appear that the market will self correct. Instead, the only way that such practices will cease and desist is for enough consumers to become aware of these practices either through WOM or initial ability to detect such practices. If this does not occur, then it may be necessary to institute additional measures to improve the truthfulness of the market.

There are several limitations to the present study that also represent opportunities for future research. First, in order to maintain model tractability and focus on the most relevant factors associated with false promotions, we do not model quality uncertainty, and we rule out the possibility for consumers to infer product quality from the list price. If we were to allow for quality uncertainty and consumer learning from the list price, then the probability for a false promotion might even be higher. We also make specific assumptions on the functional forms for the length of the Hotelling line and the consumer's utility function. We assert that neither of these assumptions is necessary for our general results. 
Second, we do not explicitly model the consumer information acquisition process, but instead assume an exogenous initial size of sophisticated segment and a fixed WOM effect over time. One extension would be to allow for endogenous information acquisition (e.g., Liu, 2011) and heterogeneous learning cost, thereby endogenizing the size of the sophisticated segment. This could also lead to interesting consumer welfare implications.

Finally, we simplify the model by not recognizing any increased consumption (and thus increased purchase volume) associated with promotions. If consumption increases under promotions, then it will make false promotions more desirable for the firms and strengthen our results.

With all this noted, we have been able to show our basic conclusions hold qualitatively using two other utility specifications that reflect reference-dependence and for different rules of the game in terms of who moves when. We find this robustness to our underlying assumptions comforting. 


\section{References}

Anderson, Eric T., Duncan I. Simester. 1998. The role of sale signs. Marketing Science 17(2) $139-155$.

Anderson, Eric T., Duncan I. Simester. 2001. Are sale signs less effective when more products have them? Marketing Science 20(2) 121-142.

Banerjee, Abhijit, Drew Fudenberg. 2004. Word-of-mouth learning. Games and Economic Behavior 46(1) $1-22$.

Board, Simon, Moritz Meyer-ter-Vehn. 2013. Reputation for quality. Econometrica 81(6) 23812462.

Breiter, Hans C., Itzhak Aharon, Daniel Kahneman, Anders Dale, Peter Shizgal. 2001. Functional Imaging of Neural Responses to Expectancy and Experience of Monetary Gains and Losses. Neuron 30(2) 619-639.

Cabral, LuÃs, Ali Hortaçsu. 2010. The dynamics of seller reputation: evidence from ebay*. The Journal of Industrial Economics 58(1) 54-78.

Chintagunta, Pradeep K. 1992. Estimating a multinomial probit model of brand choice using the method of simulated moments. Marketing Science 11(4) 386-407.

Durant, T Clark, Michael Weintraub, Daniel Houser, Shuwen Li. Forthcoming. Trust in the executive: Requiring consensus and turn-taking in the experimental lab. Journal of Peace Research

Ellison, Glenn. 2006. Bounded rationality in industrial organization. Cambridge University Press, Cambridge, 142-174.

Ellison, Glenn, Drew Fudenberg. 1995. Word-of-mouth communication and social learning. The Quarterly Journal of Economics 110(1) 93-125.

Hardie, Bruce G. S., Eric J. Johnson, Peter S. Fader. 1993. Modeling Loss Aversion and Reference Dependence Effects on Brand Choice. Marketing Science 12(4) 378-394. 
Heidhues, Paul, Botond Köszegi. 2008. Competition and Price Variation When Consumers Are Loss Averse. American Economic Review 98(4) 1245-1268.

Heidhues, Paul, Botond Köszegi. 2014. Regular prices and sales. Theoretical Economics 9(1) $217-251$.

Inman, J Jeffrey, Leigh McAlister. 1993. A retailer promotion policy model considering promotion signal sensitivity. Marketing Science 12(4) 339-356.

Kahneman, Daniel, Amos Tversky. 1979. Prospect theory: An analysis of decision under risk. Econometrica 47(2) 263-291.

Köszegi, Botond, Matthew Rabin. 2006. A Model of Reference-Dependent Preferences. The Quarterly Journal of Economics 121(4) 1133-1165.

Kováč, Eugen, Robert C. Schmidt. 2014. Market share dynamics in a duopoly model with wordof-mouth communication. Games and Economic Behavior 83 178-206.

Lien, Jaimie W., Jie Zheng. 2015. Deciding When to Quit: Reference-Dependence over Slot Machine Outcomes. The American Economic Review 105(5) 366-370.

Lindsey-Mullikin, Joan, Ross D Petty. 2011. Marketing tactics discouraging price search: deception and competition. Journal of Business Research 64(1) 67-73.

Liu, Qingmin. 2011. Information acquisition and reputation dynamics. The Review of Economic Studies 78(4) 1400-1425.

Ray, Debajyoti, Matthew Shum, Colin F. Camerer. 2015. Loss Aversion in Post-Sale Purchases of Consumer Products and their Substitutes. The American Economic Review 105(5) 376-380.

Rob, Rafael, Arthur Fishman. 2005. Is Bigger Better? Customer Base Expansion through WordâofâMouth Reputation. Journal of Political Economy 113(5) 1146-1162.

Streitfeld, David. 2016. It's discounted, but is it a deal? how list prices lost their meaning. The New York Times .

Taylor, Curtis R. 2004. Consumer privacy and the market for customer information. The RAND Journal of Economics 35(4) 631-650. 
Thaler, Richard. 1985. Mental accounting and consumer choice. Marketing Science 4(3) 199-214.

Tversky, Amos, Daniel Kahneman. 1991. Loss Aversion in Riskless Choice: A Reference-Dependent Model. The Quarterly Journal of Economics 106(4) 1039-1061.

Weber, Bernd, Andreas Aholt, Carolin Neuhaus, Peter Trautner, Christian E. Elger, Thorsten Teichert. 2007. Neural evidence for reference-dependence in real-market-transactions. NeuroImage 35(1) 441-447.

Zhang, Mu, Jie Zheng. 2017. A robust reference-dependent model for speculative bubbles. Journal of Economic Behavior $\&$ Organization 137 232-258. 


\section{Appendix}

\section{A Proofs}

\section{Proof of Proposition 1}

We have proved cases under $\{N, N\},\{T, T\}$ and $\{F, F\}$ in the main paper. Below we prove the rest cases.

$\{T, N\}$ : When the promotion strategy profile is $\{T, N\}$, store 2 charges the regular price $p^{\star}$ and store 1 offers a real promotion with discount rate $\alpha_{1}^{T N}$. If consumer $x$ purchases from store 1 , then her utility is $u_{1}(x)=\left(1+\alpha_{1}^{T N}\right) v-\left(1-\alpha_{1}^{T N}\right) p^{\star}-x$. If she purchases from store 2 , then her utility is $u_{2}(x)=v-p^{\star}-\left[\left(v+p^{\star}\right) d-x\right]$. The consumer who is indifferent between purchasing from the two stores is located at $x^{T N}=\frac{\left(v+p^{\star}\right)\left(d+\alpha_{1}^{T N}\right)}{2}$. The normalized payoff of store 1 is $\frac{\left(1-\alpha_{1}^{T N}\right)\left(d+\alpha_{1}^{T N}\right)}{d}$, leading to store 1's optimal discount rate $\alpha_{1}^{T N}=\frac{1-d}{2}$. Store 1's demand is in turn $\left(\frac{1+d}{4}\right)\left(v+p^{\star}\right)$ and store 2 's demand is $\left(\frac{3 d-1}{4}\right)\left(v+p^{\star}\right)$. The normalized payoffs of store 1 and 2 are $\left(\frac{(1+d)^{2}}{4 d}, \frac{3 d-1}{2 d}\right)$. $u_{i}>0(i=1,2)$ requires $\frac{v}{p^{\star}}>\frac{3+3 d}{5-3 d}$ and is satisfied under our assumption.

$\{N, T\}$ : When the promotion strategy profile is $\{N, T\}$, the problem is symmetric to $\{T, N\}$.

$\{N, F\}$ : When the promotion strategy profile is $\{N, F\}$, store 1 charges the regular price $p^{\star}$ and store 2 has a false promotion with discount rate $\alpha^{N F}$. Because store 2 mimics the discount rate under strategy profile $\{N, T\}, \alpha^{N F}=\alpha^{N T}=\frac{1-d}{2}$. If a sophisticated consumer $x$ purchases from store 1 , then her utility is $u_{s 1}(x)=v-p^{\star}-x$. If she purchases from store 2 , then her utility is $u_{s 2}(x)=\left(1-\alpha^{N F}\right) v-p^{\star}-\left[\left(v+p^{\star}\right) d-x\right]$. The sophisticated consumer who is indifferent between purchasing from the two stores is located at $x_{s}^{N F}=\frac{\left(v+p^{\star}\right) d+\alpha^{N F} v}{2}$. If a naive consumer $x$ purchases from store 1 , then her utility is $u_{n 1}(x)=v-p^{\star}-x$. If she purchases from store 2 , then her utility is $u_{n 2}(x)=\left(1+\alpha^{N F}\right) v-p^{\star}-\left[\left(v+p^{\star}\right) d-x\right]$. The naive consumer who is indifferent between purchasing from the two stores is located at $x_{n}^{N F}=\frac{\left(v+p^{\star}\right) d-\alpha^{N F} v}{2}$. Taken together, the demand of store 1 is $\tilde{\lambda} \frac{\left(v+p^{\star}\right) d+\alpha^{N F} v}{2}+(1-\tilde{\lambda}) \frac{\left(v+p^{\star}\right) d-\alpha^{N F} v}{2}$, and the demand of store 2 is $\tilde{\lambda} \frac{\left(v+p^{\star}\right) d-\alpha^{N F} v}{2}+(1-\tilde{\lambda}) \frac{\left(v+p^{\star}\right) d+\alpha^{N F} v}{2}$. $\tilde{\lambda}$ is the population of sophisticated consumers. In period $1, \tilde{\lambda}=\lambda$. In period $2, \tilde{\lambda}=\lambda$ if no store offered false promotions in period 1 , and $\tilde{\lambda}=\lambda+\epsilon$ if false promotions occurred in period 1. Therefore, the normalized payoffs of store 1 and 2 are 
$\left(1+\frac{(1-d)(2 \tilde{\lambda}-1) v}{2\left(v+p^{\star}\right) d}, 1-\frac{(1-d)(2 \tilde{\lambda}-1) v}{2\left(v+p^{\star}\right) d}\right) \cdot u_{s i}>0(i=1,2)$ requires $\frac{v}{p^{\star}}>\frac{4+2 d}{3-d}$ and is satisfied under our assumption. $u_{n i}>0(i=1,2)$ requires $\frac{v}{p^{\star}}>\frac{4+2 d}{5-3 d}$ and is also satisfied under our assumption.

$\{F, N\}$ : When the promotion strategy profile is $\{F, N\}$, the problem is symmetric to $\{N, F\}$.

$\{T, F\}$ : When the promotion strategy profile is $\{T, F\}$, store 1 has a real promotion with discount rate $\alpha_{1}^{T F}$ and store 2 has a false promotion with discount rate $\alpha_{2}^{T F}$. Because store 2 will mimic the discount rate under strategy profile $\{T, T\}, \alpha_{2}^{T F}=\alpha_{2}^{T T}=1-d$. If a sophisticated consumer $x$ purchases from store 1 , then her utility is $u_{s 1}(x)=\left(1+\alpha_{1}^{T F}\right) v-\left(1-\alpha_{1}^{T F}\right) p^{\star}-x$. If she purchases from store 2 , then her utility is $u_{s 2}(x)=\left(1-\alpha_{2}^{T F}\right) v-p^{\star}-\left[\left(v+p^{\star}\right) d-x\right]$.The sophisticated consumer who is indifferent between purchasing from the two stores is located at $x_{s}^{T F}=\frac{\left(p^{\star}+v\right)\left(d+\alpha_{1}^{T F}\right)+\alpha_{2}^{T F} v}{2}$. Likewise, the naive consumer who is indifferent between purchasing from the two stores is located at $x_{n}^{T F}=\frac{\left(p^{\star}+v\right)\left(d+\alpha_{1}^{T F}\right)-\alpha_{2}^{T F} v}{2}$. Taken together, the demand of store 1 is $\tilde{\lambda} \frac{\left(p^{\star}+v\right)\left(d+\alpha_{1}^{T F}\right)+\alpha_{2}^{T F} v}{2}+(1-\tilde{\lambda}) \frac{\left(p^{\star}+v\right)\left(d+\alpha_{1}^{T F}\right)-\alpha_{2}^{T F} v}{2}$, and the demand of store 2 is $\tilde{\lambda} \frac{\left(p^{\star}+v\right)\left(d-\alpha_{1}^{T F}\right)-\alpha_{2}^{T F} v}{2}+(1-\tilde{\lambda}) \frac{\left(p^{\star}+v\right)\left(d-\alpha_{1}^{T F}\right)+\alpha_{2}^{T F} v}{2}$. Hence, the normalized payoff of store 1 is $\frac{\left(1-\alpha_{1}^{T F}\right)\left[\left(p^{\star}+v\right)\left(d-\alpha_{1}^{T F}\right)+\alpha_{2}^{T F}(2 \tilde{\lambda}-1) v\right]}{\left(p^{\star}+v\right) d}$, and the optimal discount of store 1 is in turn $\alpha_{1}^{\star}(\tilde{\lambda})=$ $\frac{(1-d)\left[p^{\star}+2(1-\tilde{\lambda}) v\right]}{2\left(p^{\star}+v\right)}$. It is straightforward to show that $\alpha_{1}^{\star}(\tilde{\lambda})<1-d$. Therefore, the normalized payoff of store 1 and 2 is represented as $\left(\left(p^{\star}+v\right)\left(\frac{1-\alpha_{1}^{\star}(\tilde{\lambda})}{2}\right),\left(p^{\star}+v\right)\left(d-\frac{1-\alpha_{1}^{\star}(\tilde{\lambda})}{2}\right)\right)$. Substituting $\alpha_{1}^{\star}(\tilde{\lambda})$ into the formula, the normalized payoffs are $\left(\frac{\left(1-\alpha_{1}^{\star}(\tilde{\lambda})\right)^{2}}{d}, 2-\frac{1-\alpha_{1}^{\star}(\tilde{\lambda})}{d}\right) \cdot u_{s i}>0$ $(i=1,2)$ requires $\frac{v}{p^{\star}}>\frac{1+2 d}{2-d}$ and is satisfied under our assumption. $u_{n i}>0(i=1,2)$ requires $\frac{v}{p^{\star}}>\frac{1+2 d}{4-3 d}$ and is also satisfied under our assumption.

$\{F, T\}$ : When the promotion strategy profile is $\{F, T\}$, the problem is symmetric to $\{T, F\}$.

\section{Proof of Lemma 2}

Step 1. Define $f(\theta)=\pi_{1}(\theta)-\pi^{F F}(\theta)$. It is straightforward to show that $f(\theta)$ is strictly increasing with $f(0)=0$. Notice that

$$
\pi_{1}(1)=\frac{1}{d}\left[\frac{\frac{1+d}{2}+\frac{v}{p^{\star}}}{1+\frac{v}{p^{\star}}}\right]^{2}>\frac{(1+d)^{2}}{4 d}>1 .
$$


Evaluating $f(\theta)$ at $\theta=1$, we have:

$$
\begin{aligned}
f(1) & =\pi_{1}(1)-\pi^{F F}(1) \\
& >1-\pi^{F F}(1)>0,
\end{aligned}
$$

where the first inequality follows the fact that $\pi_{1}(1)>1$ and the second inequality follows $\pi^{F F}<1$. Therefore, $\underline{\theta} \in(0,1)$.

Step 2. With a little abuse of notation, we define $g(x)=\pi_{1}(x)-\pi^{F F}(x)$, where $x=\frac{v}{p^{\star}}$, as a function of $\frac{v}{p^{\star}}$. It is straightforward to show that $g(x)$ is strictly decreasing. Consequently, we have $\underline{\theta} \in\left(\underline{\theta}_{\star}, \underline{\theta}^{\star}\right)$, where $\underline{\theta}^{\star}$ and $\underline{\theta}_{\star}$ satisfy the following equations:

$$
\pi_{1}\left(\underline{\theta}^{\star} \mid \frac{v}{p^{\star}}=\frac{2+d}{d}\right)=\pi^{F F}\left(\underline{\theta}^{\star} \mid \frac{v}{p^{\star}}=\frac{2+d}{d}\right)
$$

and

$$
\pi_{1}\left(\underline{\theta}_{\star} \mid \frac{v}{p^{\star}}=\frac{4+2 d}{3-d}\right)=\pi^{F F}\left(\underline{\theta}_{\star} \mid \frac{v}{p^{\star}}=\frac{4+2 d}{3-d}\right),
$$

respectively. Simplifying the first equation yields the closed-form expression of $\underline{\theta}^{\star}$.

Step 3. Finally, we need to show $\underline{\theta}^{\star}<\frac{1}{2}$. To this end, we have

$$
\begin{aligned}
\underline{\theta}^{\star}-\frac{1}{2} & =\frac{p^{\star}+v}{2 v}\left[2 \frac{\sqrt{d}-d}{1-d}-1\right] \\
& =\frac{p^{\star}+v}{2 v(1-d)}[2 \sqrt{d}-1-d] \\
& =-\frac{\left(p^{\star}+v\right)(1-\sqrt{d})^{2}}{2 v(1-d)}<0 .
\end{aligned}
$$

This completes the proof.

\section{Proof of Proposition 3}

Notice first that $\bar{\theta} \leq \underline{\theta}$ cannot happen if $d>\frac{3-\sqrt{5}}{2}$. This is because $\bar{\theta}>\underline{\theta}^{\star}$ in this case. In contrast, if $d \in\left(\frac{1}{3}, \frac{3-\sqrt{5}}{2}\right), \underline{\theta}$ might be greater than $\bar{\theta}$ because $\bar{\theta}<\underline{\theta}^{\star}$. There are two different scenarios. On the one hand, $\bar{\theta} \leq \underline{\theta}$ if $\bar{\theta}$ is smaller than the lower bound of $\underline{\theta}$ regardless of the value of $\frac{v}{p^{\star}}$. On the other hand, if $\bar{\theta}>\underline{\theta}_{\star}$, then since $\bar{\theta}<\underline{\theta}^{\star}$, by continuity, it must be the case that there is a 
$\frac{\hat{v}}{p^{\star}} \in\left(\frac{4+2 d}{3-d}, \frac{2+d}{d}\right)$ such that $\bar{\theta} \leq \underline{\theta}$ if $\frac{v}{p^{\star}} \in\left(\frac{\hat{v}}{p^{\star}}, \frac{2+d}{d}\right)$ and $\bar{\theta}>\underline{\theta}$ if $\frac{v}{p^{\star}} \in\left(\frac{4+2 d}{3-d}, \frac{\hat{v}}{p^{\star}}\right)$. This completes the proof.

\section{B Derivation of Table 2}

Case $1 \lambda+\epsilon<\min \{\underline{\theta}, \bar{\theta}\}$

Discussed in Proposition 4.

Case $2 \lambda<\underline{\theta}<\lambda+\epsilon<\bar{\theta}$

There are two Nash equilibrium in the second period when $\mu^{\prime}=1:\{T, F\}$ and $\{F, T\}$.

If the Nash equilibrium in the second period is $\{T, F\}$ when $\mu^{\prime}=1$ and $\{F, F\}$ when $\mu^{\prime}=\mu$. The forward looking payoff matrix in the first period is therefore as follows.

Store 2

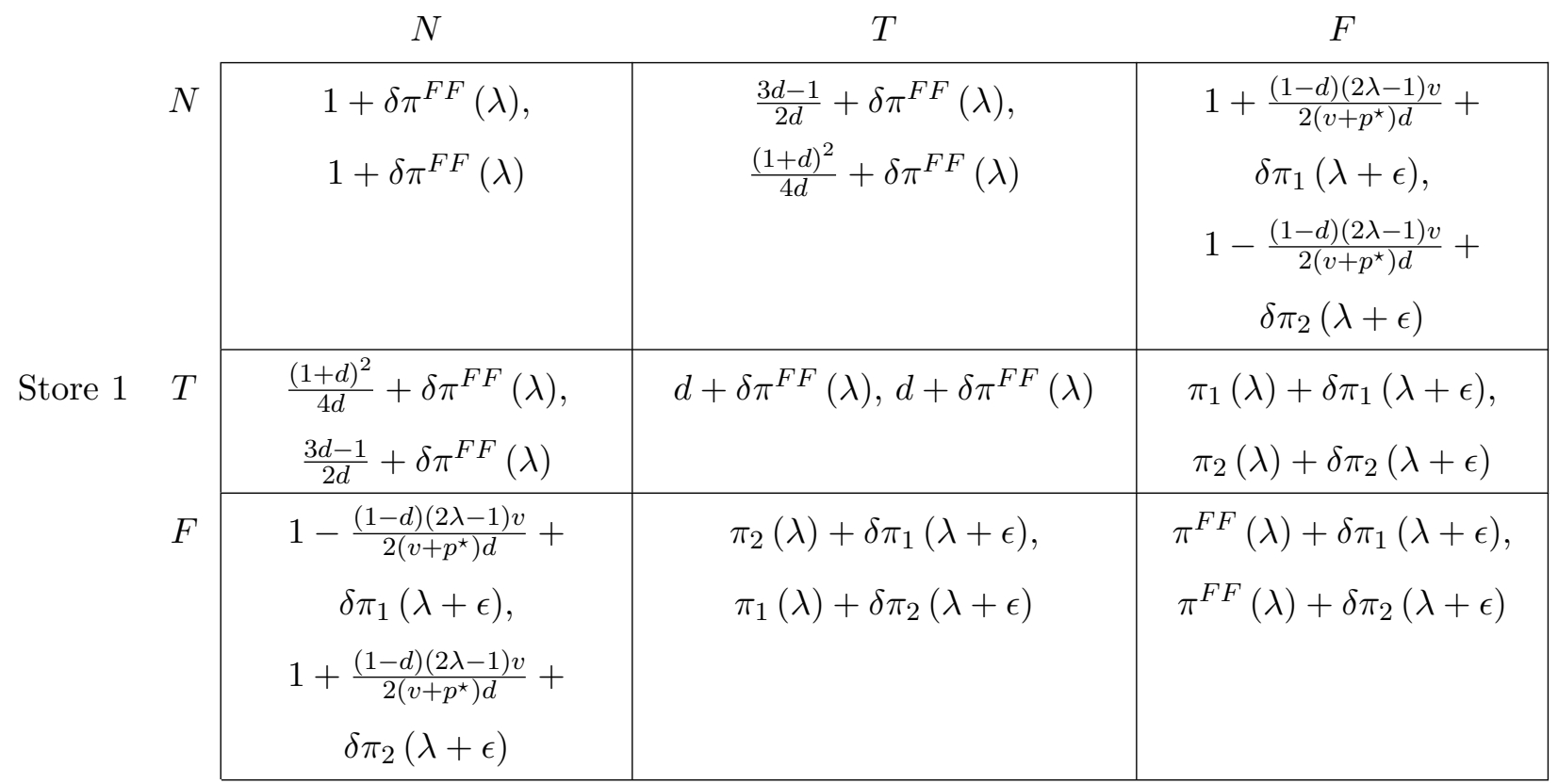

$\{F, F\}$ is always a Nash equilibrium. In this case, $\mu^{\prime}=1$. Hence, the corresponding SPNE is $\{\{F, F\},\{T, F\}\}$. In addition, if

$$
d+\delta \pi^{F F}(\lambda)>\pi_{2}(\lambda)+\delta \pi_{1}(\lambda+\epsilon)
$$

there is another Nash equilibrium $\{T, T\}$. This condition can be simplified as $\epsilon<\bar{\epsilon}_{2}$. In this case, $\mu^{\prime}=\mu$. Hence, the corresponding SPNE is $\{\{T, T\},\{F, F\}\}$. 
If the Nash equilibrium in the second period is $\{F, T\}$ when $\mu^{\prime}=1$ and $\{F, F\}$ when $\mu^{\prime}=\mu$. The forward looking payoff matrix in the first period is therefore as follows.

Store 2

\begin{tabular}{|c|c|c|c|c|}
\hline \multirow{4}{*}{ Store 1} & \multicolumn{2}{|r|}{$N$} & $T$ & $F$ \\
\hline & $N$ & $1+\delta \pi^{F F}(\lambda), 1+\delta \pi^{F F}(\lambda)$ & $\begin{array}{l}\frac{3 d-1}{2 d}+\delta \pi^{F F}(\lambda) \\
\frac{(1+d)^{2}}{4 d}+\delta \pi^{F F}(\lambda)\end{array}$ & $\begin{array}{l}1+\frac{(1-d)(2 \lambda-1) v}{2\left(v+p^{\star}\right) d}+\delta \pi_{2}(\lambda+\epsilon), \\
1-\frac{(1-d)(2 \lambda-1) v}{2\left(v+p^{\star}\right) d}+\delta \pi_{1}(\lambda+\epsilon)\end{array}$ \\
\hline & $T$ & $\begin{array}{l}\frac{(1+d)^{2}}{4 d}+\delta \pi^{F F}(\lambda) \\
\frac{3 d-1}{2 d}+\delta \pi^{F F}(\lambda)\end{array}$ & $d+\delta \pi^{F F}(\lambda), d+\delta \pi^{F F}(\lambda)$ & $\begin{array}{l}\pi_{1}(\lambda)+\delta \pi_{2}(\lambda+\epsilon) \\
\pi_{2}(\lambda)+\delta \pi_{1}(\lambda+\epsilon)\end{array}$ \\
\hline & $F$ & $\begin{array}{l}1-\frac{(1-d)(2 \lambda-1) v}{2\left(v+p^{\star}\right) d}+\delta \pi_{2}(\lambda+\epsilon) \\
1+\frac{(1-d)(2 \lambda-1) v}{2\left(v+p^{\star}\right) d}+\delta \pi_{1}(\lambda+\epsilon)\end{array}$ & $\begin{array}{l}\pi_{2}(\lambda)+\delta \pi_{2}(\lambda+\epsilon) \\
\pi_{1}(\lambda)+\delta \pi_{1}(\lambda+\epsilon)\end{array}$ & $\begin{array}{l}\pi^{F F}(\lambda)+\delta \pi_{2}(\lambda+\epsilon) \\
\pi^{F F}(\lambda)+\delta \pi_{1}(\lambda+\epsilon)\end{array}$ \\
\hline
\end{tabular}

$\{F, F\}$ is always a Nash equilibrium. In this case, $\mu^{\prime}=1$. Hence, the corresponding SPNE is $\{\{F, F\},\{F, T\}\}$. In addition, if

$$
d+\delta \pi^{F F}(\lambda)>\pi_{2}(\lambda)+\delta \pi_{1}(\lambda+\epsilon)
$$

there is another Nash equilibrium $\{T, T\}$. This condition can be simplified as $\epsilon<\bar{\epsilon}_{2}$. In this case, $\mu^{\prime}=\mu$. Hence, the corresponding SPNE is $\{\{T, T\},\{F, F\}\}$.

In this case, if $F$ occurred in the first period, $\{F, F\}$ is no longer a Nash equilibrium in the second period due to the expansion of the sophisticated segment. If $F$ does not occur in the first period, then $\{F, F\}$ can be a Nash equilibrium in the second period if the effect of WOM is within a specific range.

Case $3 \lambda<\bar{\theta}<\lambda+\epsilon<\underline{\theta}$

There are two Nash equilibria in the second period when $\mu^{\prime}=1:\{T, T\}$ and $\{F, F\}$.

If the Nash equilibrium in the second period is $\{T, T\}$ when $\mu^{\prime}=1$ and $\{F, F\}$ when $\mu^{\prime}=\mu$. The forward looking payoff matrix in the first period is therefore as follows. 
Store 2

\begin{tabular}{|c|c|c|c|c|}
\hline \multirow{4}{*}{ Store 1} & \multicolumn{2}{|r|}{$N$} & $T$ & $F$ \\
\hline & $N$ & $1+\delta \pi^{F F}(\lambda), 1+\delta \pi^{F F}(\lambda)$ & $\begin{array}{l}\frac{3 d-1}{2 d}+\delta \pi^{F F}(\lambda) \\
\frac{(1+d)^{2}}{4 d}+\delta \pi^{F F}(\lambda)\end{array}$ & $\begin{array}{l}1+\frac{(1-d)(2 \lambda-1) v}{2\left(v+p^{\star}\right) d}+\delta d \\
1-\frac{(1-d)(2 \lambda-1) v}{2\left(v+p^{\star}\right) d}+\delta d\end{array}$ \\
\hline & $T$ & $\begin{array}{c}\frac{(1+d)^{2}}{4 d}+\delta \pi^{F F}(\lambda) \\
\frac{3 d-1}{2 d}+\delta \pi^{F F}(\lambda)\end{array}$ & $d+\delta \pi^{F F}(\lambda), d+\delta \pi^{F F}(\lambda)$ & $\pi_{1}(\lambda)+\delta d, \pi_{2}(\lambda)+\delta d$ \\
\hline & $F$ & $\begin{array}{l}1-\frac{(1-d)(2 \lambda-1) v}{2\left(v+p^{\star}\right) d}+\delta d \\
1+\frac{(1-d)(2 \lambda-1) v}{2\left(v+p^{\star}\right) d}+\delta d\end{array}$ & $\pi_{2}(\lambda)+\delta d, \pi_{1}(\lambda)+\delta d$ & $\pi^{F F}(\lambda)+\delta d, \pi^{F F}(\lambda)+\delta d$ \\
\hline
\end{tabular}

$\{F, F\}$ is always a Nash equilibrium. In this case, $\mu^{\prime}=1$. Hence, the corresponding SPNE is $\{\{F, F\},\{T, T\}\}$. In addition, there is another Nash equilibrium $\{T, T\}$. In this case, $\mu^{\prime}=\mu$. Hence, the corresponding SPNE is $\{\{T, T\},\{F, F\}\}$.

If the Nash equilibrium in the second period is $\{F, F\}$ when $\mu^{\prime}=1$ and $\{F, F\}$ when $\mu^{\prime}=\mu$. The forward looking payoff matrix in the first period is therefore as follows.

Store 2

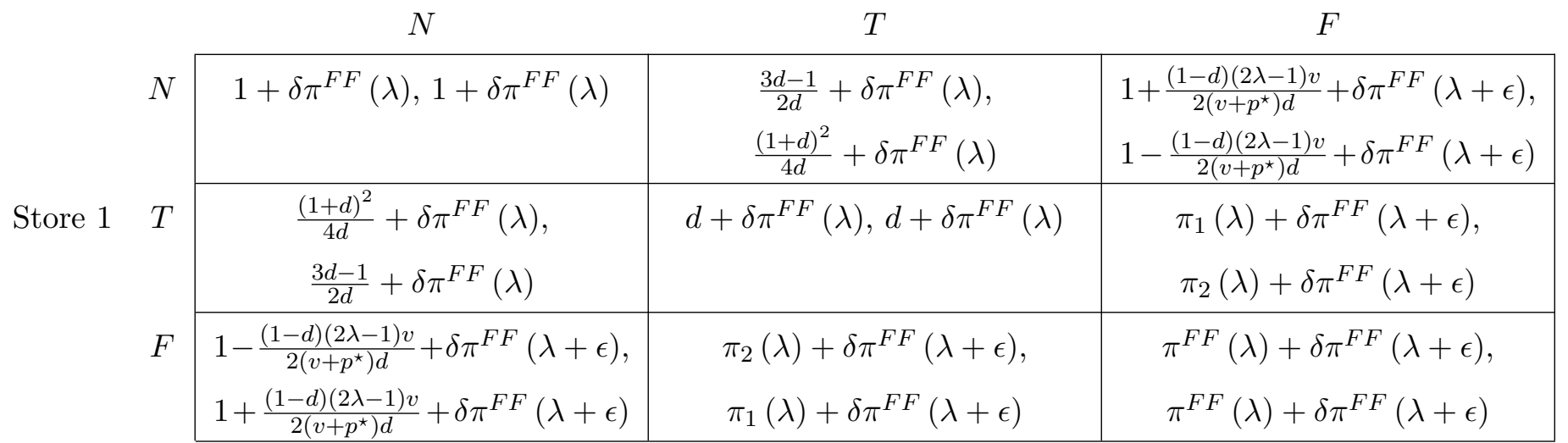

$\{F, F\}$ is always a Nash equilibrium. In this case, $\mu^{\prime}=1$. Hence, the corresponding SPNE is $\{\{F, F\},\{F, F\}\}$. In addition, if

$$
d+\delta \pi^{F F}(\lambda)>\pi_{2}(\lambda)+\delta \pi^{F F}(\lambda+\epsilon)
$$

there is another Nash equilibrium $\{T, T\}$. This condition can be simplified as $\epsilon>\underline{\epsilon}_{1}$. In this case, $\mu^{\prime}=\mu$. Hence, the corresponding SPNE is $\{\{T, T\},\{F, F\}\}$. 
If $F$ does not occur in the first period, the situation is similar to the previous case. If $F$ occurs in the first period, then in the second period, the fraction of sophisticated consumers is between $\bar{\theta}$ and $\underline{\theta}$, hence different from the case where $\underline{\theta}<\bar{\theta}$, firms will adopt symmetric strategies in the second period, leading to different SPNEs.

Case $4 \underline{\theta}<\lambda<\lambda+\epsilon<\bar{\theta}$

In this case, there are two Nash equilibria, $\{T, F\}$ and $\{F, T\}$, in the second period for both $\mu^{\prime}=\mu$ and $\mu^{\prime}=1$.

Scenario 1. If the Nash equilibrium in the second period is $\{T, F\}$ when $\mu^{\prime}=1$ and $\{T, F\}$ when $\mu^{\prime}=\mu$. The forward looking payoff matrix in the first period is therefore as follows.

Store 2

\begin{tabular}{|c|c|c|c|c|}
\hline \multirow{4}{*}{ Store 1} & & $N$ & $T$ & $F$ \\
\hline & $N$ & $1+\delta \pi_{1}(\lambda), 1+\delta \pi_{2}(\lambda)$ & $\begin{array}{l}\frac{3 d-1}{2 d}+\delta \pi_{1}(\lambda) \\
\frac{(1+d)^{2}}{4 d}+\delta \pi_{2}(\lambda)\end{array}$ & $\begin{array}{l}1+\frac{(1-d)(2 \lambda-1) v}{2\left(v+p^{\star}\right) d}+\delta \pi_{1}(\lambda+\epsilon) \\
1-\frac{(1-d)(2 \lambda-1) v}{2\left(v+p^{\star}\right) d}+\delta \pi_{2}(\lambda+\epsilon)\end{array}$ \\
\hline & $T$ & $\begin{array}{l}\frac{(1+d)^{2}}{4 d}+\delta \pi_{1}(\lambda) \\
\frac{3 d-1}{2 d}+\delta \pi_{2}(\lambda)\end{array}$ & $d+\delta \pi_{1}(\lambda), d+\delta \pi_{2}(\lambda)$ & $\begin{array}{l}\pi_{1}(\lambda)+\delta \pi_{1}(\lambda+\epsilon) \\
\pi_{2}(\lambda)+\delta \pi_{2}(\lambda+\epsilon)\end{array}$ \\
\hline & $F$ & $\begin{array}{l}1-\frac{(1-d)(2 \lambda-1) v}{2\left(v+p^{\star}\right) d}+\delta \pi_{1}(\lambda+\epsilon), \\
1+\frac{(1-d)(2 \lambda-1) v}{2\left(v+p^{\star}\right) d}+\delta \pi_{2}(\lambda+\epsilon)\end{array}$ & $\begin{array}{l}\pi_{2}(\lambda)+\delta \pi_{1}(\lambda+\epsilon) \\
\pi_{1}(\lambda)+\delta \pi_{2}(\lambda+\epsilon)\end{array}$ & $\begin{array}{l}\pi^{F F}(\lambda)+\delta \pi_{1}(\lambda+\epsilon) \\
\pi^{F F}(\lambda)+\delta \pi_{2}(\lambda+\epsilon)\end{array}$ \\
\hline
\end{tabular}

$\{F, T\}$ and $\{T, F\}$ are two Nash equilibria. In both cases, $\mu^{\prime}=1$. Hence, the corresponding SPNE are $\{\{F, T\},\{T, F\}\}$ and $\{\{T, F\},\{T, F\}\}$.

Scenario 2. If the Nash equilibrium in the second period is $\{F, T\}$ when $\mu^{\prime}=1$ and $\{T, F\}$ when $\mu^{\prime}=\mu$. The forward looking payoff matrix in the first period is therefore as follows. 
Store 2

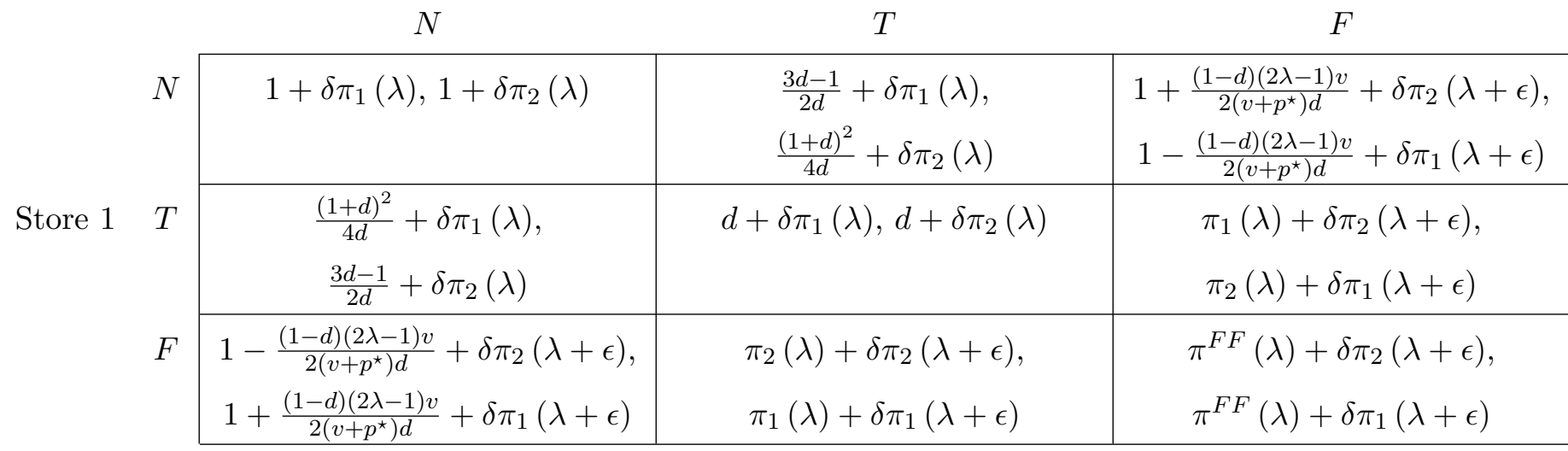

$\{T, F\}$ is a Nash equilibrium. In this cases, $\mu^{\prime}=1$. Hence, the corresponding SPNE is $\{\{T, F\},\{F, T\}\}$. In addition, if

$$
d+\delta \pi_{1}(\lambda)<\pi_{2}(\lambda)+\delta \pi_{2}(\lambda+\epsilon),
$$

there is another Nash equilibrium $\{F, T\}$. This condition can be simplified as

$$
\epsilon<\left(\frac{p^{\star}+v}{v}\right)\left(3-d-\frac{\left(\alpha^{\star}(\lambda)\right)^{2}-4 \alpha^{\star}(\lambda)}{1-d}\right)
$$

Notice that $\epsilon$ has to lie in the interval $(0, \bar{\theta}-\lambda)$. In this case, $\mu^{\prime}=1$. Hence, the corresponding SPNE is $\{\{F, T\},\{F, T\}\}$.

Scenario 3. If the Nash equilibrium in the second period is $\{F, T\}$ when $\mu^{\prime}=1$ and $\{F, T\}$ when $\mu^{\prime}=\mu$. The forward looking payoff matrix in the first period is therefore as follows.

Store 2

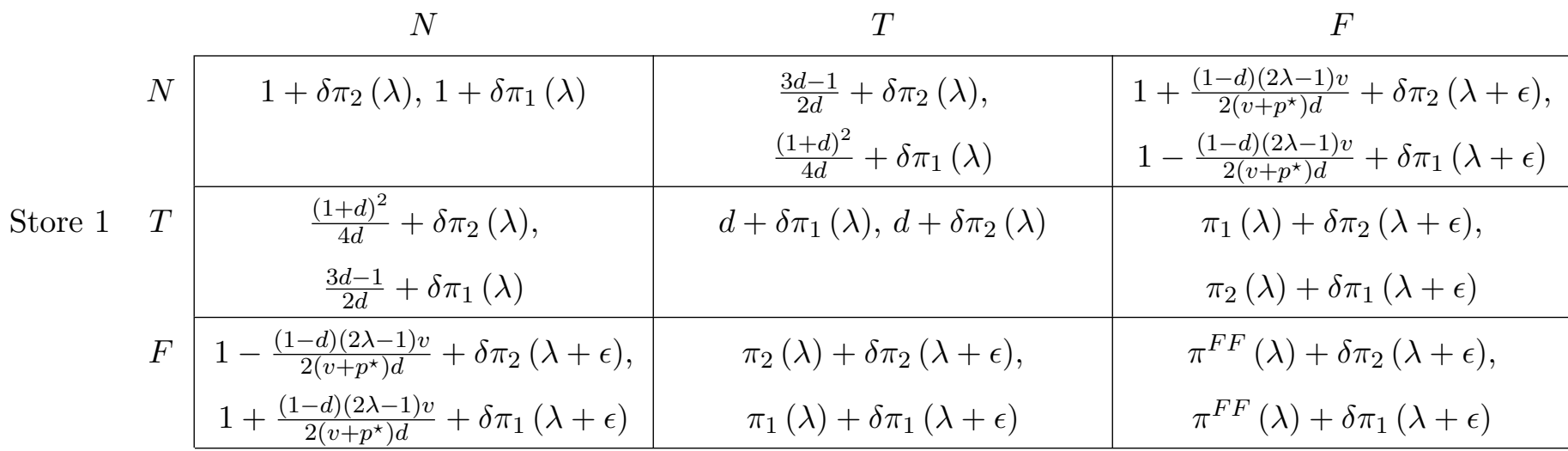


$\{F, T\}$ and $\{T, F\}$ are two Nash equilibria. In both cases, $\mu^{\prime}=1$. Hence, the corresponding SPNE are $\{\{F, T\},\{F, T\}\}$ and $\{\{T, F\},\{F, T\}\}$.

Scenario 4. If the Nash equilibrium in the second period is $\{T, F\}$ when $\mu^{\prime}=1$ and $\{F, T\}$ when $\mu^{\prime}=\mu$. The forward looking payoff matrix in the first period is therefore as follows.

Store 2

\begin{tabular}{|c|c|c|c|c|}
\hline \multirow{4}{*}{ Store 1} & \multicolumn{2}{|r|}{$N$} & $T$ & $F$ \\
\hline & $N$ & $1+\delta \pi_{2}(\lambda), 1+\delta \pi_{1}(\lambda)$ & $\begin{array}{l}\frac{3 d-1}{2 d}+\delta \pi_{2}(\lambda) \\
\frac{(1+d)^{2}}{4 d}+\delta \pi_{1}(\lambda)\end{array}$ & $\begin{array}{l}1+\frac{(1-d)(2 \lambda-1) v}{2\left(v+p^{\star}\right) d}+\delta \pi_{1}(\lambda+\epsilon) \\
1-\frac{(1-d)(2 \lambda-1) v}{2\left(v+p^{\star}\right) d}+\delta \pi_{2}(\lambda+\epsilon)\end{array}$ \\
\hline & $T$ & $\begin{array}{c}\frac{(1+d)^{2}}{4 d}+\delta \pi_{2}(\lambda) \\
\frac{3 d-1}{2 d}+\delta \pi_{1}(\lambda)\end{array}$ & $d+\delta \pi_{2}(\lambda), d+\delta \pi_{1}(\lambda)$ & $\begin{array}{c}\pi_{1}(\lambda)+\delta \pi_{1}(\lambda+\epsilon) \\
\pi_{2}(\lambda)+\delta \pi_{2}(\lambda+\epsilon)\end{array}$ \\
\hline & $F$ & $\begin{array}{l}1-\frac{(1-d)(2 \lambda-1) v}{2\left(v+p^{\star}\right) d}+\delta \pi_{1}(\lambda+\epsilon) \\
1+\frac{(1-d)(2 \lambda-1) v}{2\left(v+p^{\star}\right) d}+\delta \pi_{2}(\lambda+\epsilon)\end{array}$ & $\begin{array}{l}\pi_{2}(\lambda)+\delta \pi_{1}(\lambda+\epsilon) \\
\pi_{1}(\lambda)+\delta \pi_{2}(\lambda+\epsilon)\end{array}$ & $\begin{array}{l}\pi^{F F}(\lambda)+\delta \pi_{1}(\lambda+\epsilon) \\
\pi^{F F}(\lambda)+\delta \pi_{2}(\lambda+\epsilon)\end{array}$ \\
\hline
\end{tabular}

$\{F, T\}$ is the unique Nash equilibrium. In this cases, $\mu^{\prime}=1$. Hence, the corresponding SPNE is $\{\{F, T\},\{T, F\}\}$. In addition, if

$$
d+\delta \pi_{1}(\lambda)<\pi_{2}(\lambda)+\delta \pi_{2}(\lambda+\epsilon)
$$

there is another Nash equilibrium $\{F, T\}$. This condition can be simplified as

$$
\epsilon<\left(\frac{p^{\star}+v}{v}\right)\left(3-d-\frac{\left(\alpha^{\star}(\lambda)\right)^{2}-4 \alpha^{\star}(\lambda)}{1-d}\right)
$$

Notice that $\epsilon$ has to lie in the interval $(0, \bar{\theta}-\lambda)$. In this case, $\mu^{\prime}=1$. Hence, the corresponding SPNE is $\{\{T, F\},\{T, F\}\}$.

Similar to the previous case, if $F$ occurred in the first period, $\{F, F\}$ is no longer a Nash equilibrium in the second period due to the expansion of the sophisticated segment. However, as the initial sophisticated segment is larger in this case, $\{F, F\}$ is not a Nash equilibrium in the first period. In both periods, the fraction of sophisticated consumers is between $\underline{\theta}$ and $\bar{\theta}$, so that asymmetric strategies are adopted in both periods. 
Case $5 \bar{\theta}<\lambda<\lambda+\epsilon<\underline{\theta}$

In this case, there are two Nash equilibria, $\{T, T\}$ and $\{F, F\}$, in the second period for both $\mu^{\prime}=\mu$ and $\mu^{\prime}=1$.

Scenario 1. If the Nash equilibrium in the second period is $\{T, T\}$ when $\mu^{\prime}=1$ and $\{T, T\}$ when $\mu^{\prime}=\mu$. The forward looking payoff matrix in the first period is therefore as follows.

Store 2

\begin{tabular}{|c|c|c|c|c|}
\hline & & $N$ & $T$ & $F$ \\
\hline \multirow{3}{*}{ Store 1} & $N$ & $1+\delta d, 1+\delta d$ & $\frac{3 d-1}{2 d}+\delta d, \frac{(1+d)^{2}}{4 d}+\delta d$ & $\begin{array}{l}1+\frac{(1-d)(2 \lambda-1) v}{2\left(v+p^{\star}\right) d}+\delta d \\
1-\frac{(1-d)(2 \lambda-1) v}{2\left(v+p^{\star}\right) d}+\delta d\end{array}$ \\
\hline & $T$ & $\frac{(1+d)^{2}}{4 d}+\delta d, \frac{3 d-1}{2 d}+\delta d$ & $d+\delta d, d+\delta d$ & $\pi_{1}(\lambda)+\delta d, \pi_{2}(\lambda)+\delta d$ \\
\hline & $F$ & $\begin{array}{l}1-\frac{(1-d)(2 \lambda-1) v}{2\left(v+p^{\star}\right) d}+\delta d \\
1+\frac{(1-d)(2 \lambda-1) v}{2\left(v+p^{\star}\right) d}+\delta d\end{array}$ & $\pi_{2}(\lambda)+\delta d, \pi_{1}(\lambda)+\delta d$ & $\pi^{F F}(\lambda)+\delta d, \pi^{F F}(\lambda)+\delta d$ \\
\hline
\end{tabular}

$\{T, T\}$ and $\{F, F\}$ are two Nash equilibria. In both cases, $\mu^{\prime}=1$. Hence, the corresponding SPNE are $\{\{T, T\},\{T, T\}\}$ and $\{\{F, F\},\{T, T\}\}$.

Scenario 2. If the Nash equilibrium in the second period is $\{T, T\}$ when $\mu^{\prime}=1$ and $\{F, F\}$ when $\mu^{\prime}=\mu$. The forward looking payoff matrix in the first period is therefore as follows.

Store 2

\begin{tabular}{|c|c|c|c|c|}
\hline \multirow{4}{*}{ Store 1} & \multicolumn{2}{|r|}{$N$} & $T$ & $F$ \\
\hline & $N$ & $1+\delta \pi^{F F}(\lambda), 1+\delta \pi^{F F}(\lambda)$ & $\begin{array}{l}\frac{3 d-1}{2 d}+\delta \pi^{F F}(\lambda), \\
\frac{(1+d)^{2}}{4 d}+\delta \pi^{F F}(\lambda)\end{array}$ & $\begin{array}{l}1+\frac{(1-d)(2 \lambda-1) v}{2\left(v+p^{\star}\right) d}+\delta d \\
1-\frac{(1-d)(2 \lambda-1) v}{2\left(v+p^{\star}\right) d}+\delta d\end{array}$ \\
\hline & $T$ & $\begin{array}{c}\frac{(1+d)^{2}}{4 d}+\delta \pi^{F F}(\lambda) \\
\frac{3 d-1}{2 d}+\delta \pi^{F F}(\lambda)\end{array}$ & $d+\delta \pi^{F F}(\lambda), d+\delta \pi^{F F}(\lambda)$ & $\pi_{1}(\lambda)+\delta d, \pi_{2}(\lambda)+\delta d$ \\
\hline & $F$ & $\begin{array}{l}1-\frac{(1-d)(2 \lambda-1) v}{2\left(v+p^{\star}\right) d}+\delta d \\
1+\frac{(1-d)(2 \lambda-1) v}{2\left(v+p^{\star}\right) d}+\delta d\end{array}$ & $\pi_{2}(\lambda)+\delta d, \pi_{1}(\lambda)+\delta d$ & $\pi^{F F}(\lambda)+\delta d, \pi^{F F}(\lambda)+\delta d$ \\
\hline
\end{tabular}

$\{T, T\}$ and $\{F, F\}$ are two Nash equilibria. In this cases, $\mu^{\prime}=1$. Hence, the corresponding SPNE are $\{\{T, T\},\{T, T\}\}$ and $\{\{F, F\},\{T, T\}\}$. 
Scenario 3. If the Nash equilibrium in the second period is $\{F, F\}$ when $\mu^{\prime}=1$ and $\{T, T\}$ when $\mu^{\prime}=\mu$. The forward looking payoff matrix in the first period is therefore as follows.

Store 2

\begin{tabular}{|c|c|c|c|c|}
\hline \multirow{4}{*}{ Store 1} & & $N$ & $T$ & $F$ \\
\hline & $N$ & $1+\delta d, 1+\delta d$ & $\frac{3 d-1}{2 d}+\delta d, \frac{(1+d)^{2}}{4 d}+\delta d$ & $\begin{array}{l}1+\frac{(1-d)(2 \lambda-1) v}{2\left(v+p^{\star}\right) d}+\delta \pi^{F F}(\lambda+\epsilon), \\
1-\frac{(1-d)(2 \lambda-1) v}{2\left(v+p^{\star}\right) d}+\delta \pi^{F F}(\lambda+\epsilon)\end{array}$ \\
\hline & $T$ & $\frac{(1+d)^{2}}{4 d}+\delta d, \frac{3 d-1}{2 d}+\delta d$ & $d+\delta d, d+\delta d$ & $\begin{array}{c}\pi_{1}(\lambda)+\delta \pi^{F F}(\lambda+\epsilon) \\
\pi_{2}(\lambda)+\delta \pi^{F F}(\lambda+\epsilon)\end{array}$ \\
\hline & $F$ & $\begin{array}{l}1-\frac{(1-d)(2 \lambda-1) v}{2\left(v+p^{\star}\right) d}+\delta \pi^{F F}(\lambda+\epsilon), \\
1+\frac{(1-d)(2 \lambda-1) v}{2\left(v+p^{\star}\right) d}+\delta \pi^{F F}(\lambda+\epsilon)\end{array}$ & $\begin{array}{l}\pi_{2}(\lambda)+\delta \pi^{F F}(\lambda+\epsilon) \\
\pi_{1}(\lambda)+\delta \pi^{F F}(\lambda+\epsilon)\end{array}$ & $\begin{array}{l}\pi^{F F}(\lambda)+\delta \pi^{F F}(\lambda+\epsilon), \\
\pi^{F F}(\lambda)+\delta \pi^{F F}(\lambda+\epsilon)\end{array}$ \\
\hline
\end{tabular}

$\{F, F\}$ is a Nash equilibrium. In this cases, $\mu^{\prime}=1$. Hence, the corresponding SPNE is $\{\{F, F\},\{F, F\}\}$. In addition, if

$$
d+\delta d>\pi_{2}(\lambda)+\delta \pi^{F F}(\lambda+\epsilon)
$$

there is another Nash equilibrium $\{T, T\}$. This condition can be simplified as $\epsilon>\underline{\epsilon}_{2}$, where

$$
\underline{\epsilon}_{2}=d\left(p^{\star}+v\right)\left(\frac{\pi_{2}(\lambda)+\pi^{F F}(\lambda)-2 d}{(2+d) p^{\star}-d v}\right) .
$$

In this case, $\mu^{\prime}=1$. Hence, the corresponding SPNE is $\{\{T, T\},\{T, T\}\}$.

Scenario 4. If the Nash equilibrium in the second period is $\{F, F\}$ when $\mu^{\prime}=1$ and $\{F, F\}$ when $\mu^{\prime}=\mu$. The forward looking payoff matrix in the first period is therefore as follows. 
Store 2

\begin{tabular}{|c|c|c|c|c|}
\hline \multirow{4}{*}{ Store 1} & & $N$ & $T$ & $F$ \\
\hline & $N$ & $1+\delta \pi^{F F}(\lambda), 1+\delta \pi^{F F}(\lambda)$ & $\begin{array}{l}\frac{3 d-1}{2 d}+\delta \pi^{F F}(\lambda) \\
\frac{(1+d)^{2}}{4 d}+\delta \pi^{F F}(\lambda)\end{array}$ & $\begin{array}{l}1+\frac{(1-d)(2 \lambda-1) v}{2\left(v+p^{\star}\right) d}+\delta \pi^{F F}(\lambda+\epsilon), \\
1-\frac{(1-d)(2 \lambda-1) v}{2\left(v+p^{\star}\right) d}+\delta \pi^{F F}(\lambda+\epsilon)\end{array}$ \\
\hline & $T$ & $\begin{array}{c}\frac{(1+d)^{2}}{4 d}+\delta \pi^{F F}(\lambda), \\
\frac{3 d-1}{2 d}+\delta \pi^{F F}(\lambda)\end{array}$ & $d+\delta \pi^{F F}(\lambda), d+\delta \pi^{F F}(\lambda)$ & $\begin{array}{l}\pi_{1}(\lambda)+\delta \pi^{F F}(\lambda+\epsilon) \\
\pi_{2}(\lambda)+\delta \pi^{F F}(\lambda+\epsilon)\end{array}$ \\
\hline & $F$ & $\begin{array}{l}1-\frac{(1-d)(2 \lambda-1) v}{2\left(v+p^{\star}\right) d}+\delta \pi^{F F}(\lambda+\epsilon), \\
1+\frac{(1-d)(2 \lambda-1) v}{2\left(v+p^{\star}\right) d}+\delta \pi^{F F}(\lambda+\epsilon)\end{array}$ & $\begin{array}{l}\pi_{2}(\lambda)+\delta \pi^{F F}(\lambda+\epsilon), \\
\pi_{1}(\lambda)+\delta \pi^{F F}(\lambda+\epsilon)\end{array}$ & $\begin{array}{l}\pi^{F F}(\lambda)+\delta \pi^{F F}(\lambda+\epsilon), \\
\pi^{F F}(\lambda)+\delta \pi^{F F}(\lambda+\epsilon)\end{array}$ \\
\hline
\end{tabular}

$\{T, T\}$ and $\{F, F\}$ are two Nash equilibria. In this cases, $\mu^{\prime}=1$. Hence, the corresponding SPNE are $\{\{T, T\},\{T, T\}\}$ and $\{\{F, F\},\{T, T\}\}$.

Because in both periods, the fraction of sophisticated consumers is between $\bar{\theta}$ and $\underline{\theta}$, symmetric strategies are adopted in both periods.

Case $6 \lambda<\min \{\underline{\theta}, \bar{\theta}\}$ and $\lambda+\epsilon>\max \{\underline{\theta}, \bar{\theta}\}$

In this case, the unique Nash equilibrium in the second period is $\{T, T\}$ for $\mu^{\prime}=1$ and $\{F, F\}$ $\mu^{\prime}=\mu$. Hence, the forward looking payoff matrix in the first period is as follows.

Store 2

\begin{tabular}{|c|c|c|c|c|}
\hline \multirow{4}{*}{ Store 1} & & $N$ & $T$ & $F$ \\
\hline & $N$ & $\begin{array}{l}1+\delta \pi^{F F}(\lambda) \\
1+\delta \pi^{F F}(\lambda)\end{array}$ & $\begin{array}{l}\frac{3 d-1}{2 d}+\delta \pi^{F F}(\lambda), \\
\frac{(1+d)^{2}}{4 d}+\delta \pi^{F F}(\lambda)\end{array}$ & $\begin{array}{l}1+\frac{(1-d)(2 \lambda-1) v}{2\left(v+p^{\star}\right) d}+\delta d \\
1-\frac{(1-d)(2 \lambda-1) v}{2\left(v+p^{\star}\right) d}+\delta d\end{array}$ \\
\hline & $T$ & $\begin{array}{c}\frac{(1+d)^{2}}{4 d}+\delta \pi^{F F}(\lambda) \\
\frac{3 d-1}{2 d}+\delta \pi^{F F}(\lambda)\end{array}$ & $\begin{array}{l}d+\delta \pi^{F F}(\lambda) \\
d+\delta \pi^{F F}(\lambda)\end{array}$ & $\pi_{1}(\lambda)+\delta d, \pi_{2}(\lambda)+\delta d$ \\
\hline & $F$ & $\begin{array}{l}1-\frac{(1-d)(2 \lambda-1) v}{2\left(v+p^{\star}\right) d}+\delta d, \\
1+\frac{(1-d)(2 \lambda-1) v}{2\left(v+p^{\star}\right) d}+\delta d\end{array}$ & $\pi_{2}(\lambda)+\delta d, \pi_{1}(\lambda)+\delta d$ & $\begin{array}{l}\pi^{F F}(\lambda)+\delta d \\
\pi^{F F}(\lambda)+\delta d\end{array}$ \\
\hline
\end{tabular}

$\{F, F\}$ is one Nash equilibrium. In this case, $\mu^{\prime}=1$. Hence, the corresponding SPNE is $\{\{F, F\},\{T, T\}\}$. In addition, there is another Nash equilibrium $\{T, T\}$. In this case, $\mu^{\prime}=\mu$. Hence, the corresponding SPNE is $\{\{T, T\},\{F, F\}\}$.

In this case, the sophisticated segment is small if false promotions have never taken place, under which scenario $\{F, F\}$ is the equilibrium. However, due to the large effect of WOM, the 
sophisticated segment becomes very large once a false promotion occurs, under which scenario $\{T, T\}$ is the equilibrium.

Case $7 \underline{\theta}<\lambda<\bar{\theta}<\lambda+\epsilon$

There are two Nash equilibrium in the second period when $\mu^{\prime}=\mu:\{T, F\}$ and $\{F, T\}$.

Scenario 1. If the Nash equilibrium in the second period is $\{T, T\}$ when $\mu^{\prime}=1$ and $\{T, F\}$ when $\mu^{\prime}=\mu$. The forward looking payoff matrix in the first period is therefore as follows.

Store 2

\begin{tabular}{|c|c|c|c|c|}
\hline \multirow{4}{*}{ Store 1} & & $N$ & $T$ & $F$ \\
\hline & $N$ & $1+\delta \pi_{1}(\lambda), 1+\delta \pi_{2}(\lambda)$ & $\begin{array}{l}\frac{3 d-1}{2 d}+\delta \pi_{1}(\lambda), \\
\frac{(1+d)^{2}}{4 d}+\delta \pi_{2}(\lambda)\end{array}$ & $\begin{array}{l}1+\frac{(1-d)(2 \lambda-1) v}{2\left(v+p^{\star}\right) d}+\delta d \\
1-\frac{(1-d)(2 \lambda-1) v}{2\left(v+p^{\star}\right) d}+\delta d\end{array}$ \\
\hline & $T$ & $\begin{array}{l}\frac{(1+d)^{2}}{4 d}+\delta \pi_{1}(\lambda), \\
\frac{3 d-1}{2 d}+\delta \pi_{2}(\lambda)\end{array}$ & $d+\delta \pi_{1}(\lambda), d+\delta \pi_{2}(\lambda)$ & $\pi_{1}(\lambda)+\delta d, \pi_{2}(\lambda)+\delta d$ \\
\hline & $F$ & $\begin{array}{l}1-\frac{(1-d)(2 \lambda-1) v}{2\left(v+p^{\star}\right) d}+\delta d \\
1+\frac{(1-d)(2 \lambda-1) v}{2\left(v+p^{\star}\right) d}+\delta d\end{array}$ & $\pi_{2}(\lambda)+\delta d, \pi_{1}(\lambda)+\delta d$ & $\begin{array}{l}\pi^{F F}(\lambda)+\delta d \\
\pi^{F F}(\lambda)+\delta d\end{array}$ \\
\hline
\end{tabular}

$\{T, F\}$ is a Nash equilibrium. In this case, $\mu^{\prime}=1$. Hence, the corresponding SPNE is $\{\{T, F\},\{T, T\}\}$. In addition, there is another Nash equilibrium $\{T, T\}$. In this case, $\mu^{\prime}=\mu$. Hence, the corresponding SPNE is $\{\{T, T\},\{T, F\}\}$.

Scenario 2. If the Nash equilibrium in the second period is $\{T, T\}$ when $\mu^{\prime}=1$ and $\{F, T\}$ when $\mu^{\prime}=\mu$. The forward looking payoff matrix in the first period is therefore as follows.

Store 2

\begin{tabular}{|c|c|c|c|c|}
\hline \multirow{4}{*}{ Store 1} & \multicolumn{2}{|r|}{$N$} & $T$ & $F$ \\
\hline & $N$ & $1+\delta \pi_{2}(\lambda), 1+\delta \pi_{1}(\lambda)$ & $\begin{array}{l}\frac{3 d-1}{2 d}+\delta \pi_{2}(\lambda), \\
\frac{(1+d)^{2}}{4 d}+\delta \pi_{1}(\lambda)\end{array}$ & $\begin{array}{l}1+\frac{(1-d)(2 \lambda-1) v}{2\left(v+p^{\star}\right) d}+\delta d \\
1-\frac{(1-d)(2 \lambda-1) v}{2\left(v+p^{\star}\right) d}+\delta d\end{array}$ \\
\hline & $T$ & $\begin{array}{c}\frac{(1+d)^{2}}{4 d}+\delta \pi_{2}(\lambda) \\
\frac{3 d-1}{2 d}+\delta \pi_{1}(\lambda)\end{array}$ & $d+\delta \pi_{2}(\lambda), d+\delta \pi_{1}(\lambda)$ & $\pi_{1}(\lambda)+\delta d, \pi_{2}(\lambda)+\delta d$ \\
\hline & $F$ & $\begin{array}{l}1-\frac{(1-d)(2 \lambda-1) v}{2\left(v+p^{\star}\right) d}+\delta d \\
1+\frac{(1-d)(2 \lambda-1) v}{2\left(v+p^{\star}\right) d}+\delta d\end{array}$ & $\pi_{2}(\lambda)+\delta d, \pi_{1}(\lambda)+\delta d$ & $\begin{array}{l}\pi^{F F}(\lambda)+\delta d \\
\pi^{F F}(\lambda)+\delta d\end{array}$ \\
\hline
\end{tabular}


$\{F, T\}$ is the unique Nash equilibrium. In this case, $\mu^{\prime}=1$. Hence, the corresponding SPNE is $\{\{F, T\},\{T, T\}\}$. In addition, there is another Nash equilibrium $\{T, T\}$.In this case, $\mu^{\prime}=\mu$. Hence, the corresponding SPNE is $\{\{T, T\},\{F, T\}\}$.

Because the initial population of sophisticated consumers and the WOM effect are both large, and competition is low, it is no longer profitable for both stores to adopt strategy $F$ in either period. If false promotions have never taken place, only one store can benefit from offering a false promotion. Once the existence of false promotions is known, $\{T, T\}$ becomes the only Nash equilibrium.

Case $8 \bar{\theta}<\lambda<\underline{\theta}<\lambda+\epsilon$

There are two Nash equilibrium in the second period when $\mu^{\prime}=\mu:\{T, T\}$ and $\{F, F\}$ and a unique Nash equilibrium when $\mu^{\prime}=1:\{T, T\}$.

Scenario 1. If the Nash equilibrium in the second period is $\{T, T\}$ when both $\mu^{\prime}=1$ and $\mu^{\prime}=\mu$. The forward looking payoff matrix in the first period is therefore as follows.

Store 2

\begin{tabular}{|c|c|c|c|c|}
\hline & & $N$ & $T$ & $F$ \\
\hline \multirow{3}{*}{ Store 1} & $N$ & $1+\delta d, 1+\delta d$ & $\frac{3 d-1}{2 d}+\delta d, \frac{(1+d)^{2}}{4 d}+\delta d$ & $\begin{array}{l}1+\frac{(1-d)(2 \lambda-1) v}{2\left(v+p^{\star}\right) d}+\delta d \\
1-\frac{(1-d)(2 \lambda-1) v}{2\left(v+p^{\star}\right) d}+\delta d\end{array}$ \\
\hline & $T$ & $\frac{(1+d)^{2}}{4 d}+\delta d, \frac{3 d-1}{2 d}+\delta d$ & $d+\delta d, d+\delta d$ & $\pi_{1}(\lambda)+\delta d, \pi_{2}(\lambda)+\delta d$ \\
\hline & $F$ & $\begin{array}{l}1-\frac{(1-d)(2 \lambda-1) v}{2\left(v+p^{\star}\right) d}+\delta d \\
1+\frac{(1-d)(2 \lambda-1) v}{2\left(v+p^{\star}\right) d}+\delta d\end{array}$ & $\pi_{2}(\lambda)+\delta d, \pi_{1}(\lambda)+\delta d$ & $\pi^{F F}(\lambda)+\delta d, \pi^{F F}(\lambda)+\delta d$ \\
\hline
\end{tabular}

$\{T, T\}$ and $\{F, F\}$ are two Nash equilibria. In this cases, $\mu^{\prime}=1$. Hence, the corresponding SPNE are $\{\{T, T\},\{T, T\}\}$ and $\{\{F, F\},\{T, T\}\}$.

Scenario 2. If the Nash equilibrium in the second period is $\{T, T\}$ when $\mu^{\prime}=1$ and $\{F, F\}$ when $\mu^{\prime}=\mu$. The forward looking payoff matrix in the first period is therefore as follows. 
Store 2

\begin{tabular}{|c|c|c|c|c|}
\hline \multirow{4}{*}{ Store 1} & \multicolumn{2}{|r|}{$N$} & $T$ & $F$ \\
\hline & $N$ & $1+\delta \pi^{F F}(\lambda), 1+\delta \pi^{F F}(\lambda)$ & $\begin{array}{l}\frac{3 d-1}{2 d}+\delta \pi^{F F}(\lambda), \\
\frac{(1+d)^{2}}{4 d}+\delta \pi^{F F}(\lambda)\end{array}$ & $\begin{array}{l}1+\frac{(1-d)(2 \lambda-1) v}{2\left(v+p^{\star}\right) d}+\delta d \\
1-\frac{(1-d)(2 \lambda-1) v}{2\left(v+p^{\star}\right) d}+\delta d\end{array}$ \\
\hline & $T$ & $\begin{array}{l}\frac{(1+d)^{2}}{4 d}+\delta \pi^{F F}(\lambda), \\
\frac{3 d-1}{2 d}+\delta \pi^{F F}(\lambda)\end{array}$ & $d+\delta \pi^{F F}(\lambda), d+\delta \pi^{F F}(\lambda)$ & $\pi_{1}(\lambda)+\delta d, \pi_{2}(\lambda)+\delta d$ \\
\hline & $F$ & $\begin{array}{l}1-\frac{(1-d)(2 \lambda-1) v}{2\left(v+p^{\star}\right) d}+\delta d \\
1+\frac{(1-d)(2 \lambda-1) v}{2\left(v+p^{\star}\right) d}+\delta d\end{array}$ & $\pi_{2}(\lambda)+\delta d, \pi_{1}(\lambda)+\delta d$ & $\pi^{F F}(\lambda)+\delta d, \pi^{F F}(\lambda)+\delta d$ \\
\hline
\end{tabular}

$\{F, F\}$ is the unique Nash equilibrium. In this case, $\mu^{\prime}=1$. Hence, the corresponding SPNE is $\{\{F, F\},\{T, T\}\}$. In addition, there is another Nash equilibrium $\{T, T\}$. In this case, $\mu^{\prime}=\mu$. Hence, the corresponding SPNE is $\{\{T, T\},\{F, F\}\}$.

In this case, competition level is higher than in Case 7, so that when false promotion has not occurred, the stores will adopt symmetric strategies. Similar to Case 7, due to the large WOM effect, $\{T, T\}$ becomes the only Nash equilibrium once false promotion takes place.

Case $9 \lambda>\max \{\underline{\theta}, \bar{\theta}\}$

In this case, the unique Nash equilibrium in the second period is $\{T, T\}$ for both $\mu^{\prime}=1$ and $\mu^{\prime}=\mu$. Hence, the forward looking payoff matrix in the first period (i.e., the payoff matrix that takes into account future equilibrium outcomes) is as follows.

Store 2

\begin{tabular}{|c|c|c|c|c|}
\hline & & $N$ & $T$ & $F$ \\
\hline \multirow{3}{*}{ Store 1} & $N$ & $1+\delta d, 1+\delta d$ & $\frac{3 d-1}{2 d}+\delta d, \frac{(1+d)^{2}}{4 d}+\delta d$ & $\begin{array}{l}1+\frac{(1-d)(2 \lambda-1) v}{2\left(v+p^{\star}\right) d}+\delta d \\
1-\frac{(1-d)(2 \lambda-1) v}{2\left(v+p^{\star}\right) d}+\delta d\end{array}$ \\
\hline & $T$ & $\frac{(1+d)^{2}}{4 d}+\delta d, \frac{3 d-1}{2 d}+\delta d$ & $d+\delta d, d+\delta d$ & $\pi_{1}(\lambda)+\delta d, \pi_{2}(\lambda)+\delta d$ \\
\hline & $F$ & $\begin{array}{l}1-\frac{(1-d)(2 \lambda-1) v}{2\left(v+p^{\star}\right) d}+\delta d \\
1+\frac{(1-d)(2 \lambda-1) v}{2\left(v+p^{\star}\right) d}+\delta d\end{array}$ & $\pi_{2}(\lambda)+\delta d, \pi_{1}(\lambda)+\delta d$ & $\pi^{F F}(\lambda)+\delta d, \pi^{F F}(\lambda)+\delta d$ \\
\hline
\end{tabular}

There exists a unique Nash equilibrium $\{T, T\}$ in this game. Therefore, the belief is updated to $\mu^{\prime}=\mu$. Hence, the dynamic game has a unique $\operatorname{SPNE}\{\{T, T\},\{T, T\}\}$.

In this case, the initial population of sophisticated consumers is already extremely large. Hence, strategy $F$ cannot be viable in any period. The game boils down to the one without false promotions. 